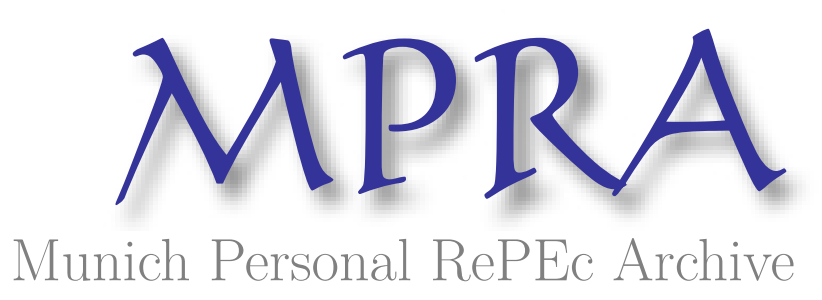

\title{
On the choice of covariance specifications for portfolio selection problems
}

R. Ferreira, Alexandre and A. P. Santos, Andre

21 August 2016

Online at https://mpra.ub.uni-muenchen.de/73259/

MPRA Paper No. 73259, posted 28 Aug 2016 20:56 UTC 


\title{
On the choice of covariance specifications for portfolio selection problems
}

\author{
Alexandre R. Ferreira*1 and André A. P. Santos ${ }^{2}$ \\ ${ }^{1} \mathrm{PhD}$ Candidate in Business, Rice University \\ ${ }^{2}$ Department of Economics, Universidade Federal de Santa Catarina
}

August 21, 2016

\begin{abstract}
Two crucial aspects to the problem of portfolio selection are the specification of the model for expected returns and their covariances, as well as the choice of the investment policy to be adopted. A common trade-off is to consider dynamic covariance specifications vis-a-vis static models such as those based on shrinkage methods. This work empirically shows that these two aspects are intrinsically attached to the impact of transaction costs. To address this question, we implement a broad range of covariance specifications to generate a set of 16 portfolio selection policies in a high dimensional sample composed by the 50 most traded stocks of the S\&P100 index. We find that GARCH-type dynamic covariances yield portfolios with superior risk-adjusted performance only in the absence of transaction costs. In more realistic scenarios involving alternative levels of transaction costs, portfolios based on static covariance models outperform. In particular, we find that a risk-averse investor with quadratic utility function is willing to pay an annualized fee of 368 basis points (bp) on average in order to switch from the dynamic covariance models to a static covariance specification when the level of transaction costs is $20 \mathrm{bp}$. Finally, portfolio policies that seek to alleviate estimation error by ignoring off-diagonal covariance elements as those proposed in Kirby \& Ostdiek (2012) are more robust specially in scenarios with higher transaction costs.
\end{abstract}

Keywords: Composite likelihood, conditional correlation models, factor models, multivariate GARCH JEL classification: C53; E43; G17.

*Corresponding author. e-mail: alexandre.ferreira@rice.edu 


\section{Introduction}

This paper is concerned with the problem of the choosing the most appropriate covariance specification for high dimensional portfolio selection and optimization. This choice is of paramount importance since the specification of the covariance matrix of asset returns is a key ingredient to many portfolio selection problems. Markowitz (1952), for instance, created the basis for the modern portfolio theory by showing the way in which variances and covariances influence portfolio risk and risk-adjusted portfolio returns. Since then, academics and market practitioners seek to enhance covariance modeling using a myriad of methods and models.

The literature points to an ample range of possible ways of modeling covariances. The most immediate choice is usually between static or dynamic models. Dynamic models are based on the idea that the current covariance depends on the covariance of previous periods, being updated according to alternative autoregressive structures such as multivariate GARCH and stochastic volatility models; see Bauwens et al. (2006) and Silvennoinen \& Teräsvirta (2009). Static models, in constrast, are those in which which there is no autoregressive covariance dynamics and, because of that, are usually more parsimonious than their dynamic counterparts. The most common static models are those in which the covariance matrix is unconditionally estimated using on a sample of asset returns or, alternatively, estimated based on a factor model that captures cross-sectional characteristics of asset returns. Among static models commonly employed in portfolio selection problems are the ones that reduce estimation error by shrinking the sample covariance matrix towards alternative targets such as those proposed in Ledoit \& Wolf (2003a,b, 2004).

To ground the decision of which type of covariance model to adopt, usually are taken into account pros and cons of each approach as, for example, ease of implementation, processing cost, as well as the ability to capture stylized facts in the covariance profile among the assets. One central aspect that must be taken into account while choosing the most appropriate covariance specification is that dynamic models usually posses the highest implementation requirements when compared to their static counterparts due to their increased parameterization. This leads to more estimation error and negatively impact portfolio performance, due to demanding more frequent portfolio re-balancing and yielding more extreme portfolio allocations, and, consequently, increasing transaction costs (Kirby \& Ostdiek, 2012). On the other hand, these models can accommodate stylized facts of financial time series such as hetoskedasticity and excess kurtosis.

In this paper we shed light on the lack of consensus in the literature regarding which is the most appropriate approach to model covariances in a realistic scenario in which i) there are many assets, ii) transaction costs are properly taken into account, and iii) there is frequent portfolio re-balancing. Our main goal is to evaluate i) how the increase in proportional transaction costs impacts portfolio performance measured by risk-adjusted returns net of transaction costs and ii) how this information can be used in order to help investors to select the most appropriate covariance specification.

Our paper is related to previous studies in the literature of covariance modeling for portfolio selection problems such as Engle \& Sheppard (2008) and Becker et al. (2014). Engle \& Sheppard (2008) evaluate 
the performance of alternative dynamic covariance specifications belonging to the multivariate GARCH family and conclude that tightly parameterized models generally perform better when evaluated with economically meaningful criteria. Becker et al. (2014) study the ability of different loss functions to discriminate between a set of competing forecasting models which are subsequently applied in a portfolio allocation context. None of the studies, however, consider the impact of transaction costs and employ only one portfolio selection policy. We add to this literature by not only taking into account the presence of transaction costs but also considering a broader set of portfolio policies.

It is also worth mentioning that the estimation of expected returns also plays its part in portfolio selection. There is evidence, however, suggesting that employing predictive models for expected returns based on autoregressive structures leads to portfolios with very high turnovers that might compromise their usage in real situations; see, for example, DeMiguel et al. (2009a) and DeMiguel et al. (2014). Because of that, the present work considers less parameterized alternatives. Specifically, we i) estimate expected returns either unconditionally via sample means or ii) assumes that a multi-factor conditional version of the capital asset pricing model (CAPM) holds, which implies that the cross-sectional variation in conditional expected excess returns is due to cross-sectional variation in conditional betas.

In our empirical exercise we implement 11 different covariance models to generate a set of 16 portfolios from different asset selection strategies in a high dimensional sample composed by the 50 most traded stocks from the S\&P100 index. We choose 3 popular static models based on shrinkage methods proposed in Ledoit \& Wolf (2004), Ledoit \& Wolf (2003a) and Ledoit \& Wolf (2003b). In short, these models shrink the sample covariance matrix, which is an unbiased estimator but more prone to estimation error, towards alternative structured covariance estimators that are biased but less prone to estimation error. In this case, the target matrices are the identity matrix, constant correlation matrix and market factors matrix, respectively. The remaining 8 specifications belong to the class of dynamic covariance models models from the multivariate GARCH family: the exponentially weighted moving average (EWMA), the optimal rolling estimator (ORE) of Foster \& Nelson (1996), the scalar VECH model of Bollerslev et al. (1988), the orthogonal GARCH (OGARCH) from Alexander (2001), the constant conditional correlation (CCC) model of Bollerslev (1990), the dynamic conditional correlation model (DCC) proposed by Engle (2002), and its asymmetric version (ASYDCC) proposed in Cappiello et al. (2006). Each of these specifications are detailed in Section 3.

The alternative covariance specifications are used to obtain a set of portfolio policies including alternative formulations of the mean-variance policy such as those that alleviate estimation risk by imposing short-sales restrictions (Jagannathan \& Ma, 2003) and norm restrictions (DeMiguel et al. , 2014). We also implement a second group of portfolio policies that aims at reducing estimation risk by ignoring off-diagonal covariance elements such as those proposed in Kirby \& Ostdiek (2012). These policies retain the most appealing features of the $1 / \mathrm{N}$ strategy extensively studied in DeMiguel et al. (2009b) (no optimization, no covariance matrix inversion, and no short sales) while exploiting sample information about the reward and risk characteristics of 
the assets under consideration.

Our results leave three most important messages. First, the choice of the covariance model is critical to the performance of a portfolio policy. For instance, we find the risk-adjusted performance measured by the Sharpe ratio can vary from -0.62 to 0.06 within the same portfolio policy by changing the covariance model used to obtain portfolio weights. Second, we find that the choice of the most appropriate covariance specification for the portfolio selection problems considered in the paper is substantially impacted by the presence and the level of transaction costs. We find that, in the absence of transaction costs, dynamic covariance models outperform static counterpars in terms of average gross returns as well as in terms of risk-adjusted returns. On average, Sharpe ratios obtained with dynamic covariance models are $12 \%$ higher than those obtained with static covariance models, and the pairwise differences are statistically significant in many instances. However, as we move to more realistic scenarios in which transaction costs are properly taken into account, we find that static covariance models clearly outperform in the vast majority of instances. Specifically, Sharpe ratios based on portfolio returns net of transaction costs of 20 basis points (bp) are $48 \%$ higher on average when static covariance models are adopted in comparison to those obtained with dynamic models. This difference in risk adjusted performance becomes even higher when transaction costs of $50 \mathrm{bp}$ are considered. A closer examination reveals that these differences in risk-adjusted performance are mainly driven by a much higher level of turnover obtained when dynamic covariance models are used to implement the portfolio policies. Finally, we find that a risk-averse investor with quadratic utility function is willing to pay an annualized fee of 368 bp on average in order to switch from the dynamic covariance models to a static covariance specification when the level of transaction costs is $20 \mathrm{bp}$.

The remainder of the document is organized this way: section 2 details the portfolio selection policies considered in the paper. Section 3 describes the dynamic and static covariance models. Section 4 discusses the methodology used to evaluate portfolio performance. Section 5 details the empirical exercise carried out. Finally, Section 6 concludes.

\section{Portfolio selection methods}

Consider an investment universe with $N$ assets $A_{1}, A_{2}, \ldots, A_{N}$ with uncertain future returns $R_{1}, R_{2}, \ldots, R_{N}$.

Let $\mathbf{R}$ be the return vector:

$$
\mathbf{R}=\left[R_{1}, R_{2}, \ldots, R_{n}\right]^{\top}
$$

The expected return vector $\boldsymbol{\mu}=E(\mathbf{R})$ contains as its elements $\mu_{i}=E\left(R_{i}\right), i=1, \ldots, n$, such that:

$$
\boldsymbol{\mu}=\left[\mu_{1}, \mu_{2}, \ldots, \mu_{n}\right]^{\top}
$$


The covariance matrix of the returns, $\Sigma=\operatorname{Var}(R)$, contains as its elements $\sigma_{i i}=\sigma_{i}^{2}$ and $\sigma_{i j}=\sigma_{j i}=\rho_{i j} \sigma_{i} \sigma_{j}$ (for $i \neq j$ ), where $\sigma_{i}$ is $R_{i}$ standard deviation and $\rho_{i j}$ is the correlation between the returns of assets $A_{i}$ and $A_{j}$ (for $i \neq j$ ). The covariance matrix $\Sigma$ is symmetric and written as:

$$
\Sigma=\left(\sigma_{i j}\right)_{i, j=1, \ldots, N}=\left[\begin{array}{cccc}
\sigma_{11} & \sigma_{12} & \cdots & \sigma_{1 n} \\
\sigma_{21} & \sigma_{22} & \cdots & \sigma_{2 n} \\
\vdots & \vdots & \ddots & \vdots \\
\sigma_{n 1} & \sigma_{n 2} & \cdots & \sigma_{n n}
\end{array}\right]
$$

All valid covariance matrices are positive semi-definite, or equivalently, all eigenvalues are non-negative. A portfolio is represented by the $N$-dimensional vector $\mathbf{w}$, such that:

$$
\mathbf{w}=\left[w_{1}, w_{2}, \ldots, w_{n}\right]^{\top}
$$

and $w_{i}$ is the share of total wealth invested in asset $A_{i}$. The portfolio return $R_{p}$ is linearly dependent of these weights and it is the weighted average of the returns of each asset involved, where the weight of each asset is the portfolio share invested in the asset, i.e.:

$$
R_{p}=w_{1} R_{1}+\cdots+w_{n} R_{n}=\sum_{i=1}^{N} w_{i} R_{i}=\mathbf{w}^{\boldsymbol{\top}} \mathbf{R} .
$$

Therefore, the expected portfolio return, $\mu_{p}$, is the weighted average of each asset expected return and the portfolio variance $\sigma_{p}^{2}$ is a quadratic function of the weight vector. We can denote these variables by:

$$
\begin{aligned}
& \mu_{p}=E\left(R_{p}\right)=E\left(\mathbf{w}^{\boldsymbol{\top}} \mathbf{R}\right)=\mathbf{w}^{\boldsymbol{\top}} \boldsymbol{\mu} \\
& \sigma_{p}^{2}=\operatorname{Var}\left(R_{p}\right)=\operatorname{Var}\left(\mathbf{w}^{\boldsymbol{\top}} \mathbf{R}\right)=\mathbf{w}^{\boldsymbol{\top}} \Sigma \mathbf{w} .
\end{aligned}
$$

\subsection{Policies}

Assume that there are $N$ risky assets with expected returns $\mu_{t}$ and covariance matrix $\Sigma_{t}$. Suppose that there is no risk free asset and that the investor need to allocate all his wealth among the $N$ risky assets. Next, we detail the portfolio selection policies considered in our empirical exercise.

Mean-variance and minimum variance. The mean-variance portfolio described by Markowitz (1952) is of paramount importance in any study about portfolio selection. To solve the mean-variance trade-off 
between risk and return the investor needs to find the weight vector that satisfies:

$$
\begin{aligned}
& \min _{w_{t}} w_{t}^{\top} \Sigma_{t} w_{t}-\frac{1}{\gamma} w_{t}^{\top} \mu_{t}, \\
& \text { s.t. } w_{t}^{\top} e=1
\end{aligned}
$$

where $\gamma>0$ represents the investor's level of relative risk aversion and $e$ is a vector of ones. $\mu_{t}=\frac{1}{T} \sum_{i=1}^{T} R$ is the vector of expected returns, where $T$ is the length of the estimation window. The specification of the covariance matrix $\Sigma_{t}$ depends on whether we are using a dynamic or a static model; see Section 3 . The component $w_{t}^{\top} \Sigma_{t} w_{t}$ represents the portfolio risk. The component $w_{t}^{\top} \mu_{t}$ represents the portfolio return. We follow DeMiguel et al. (2009a) and set $\gamma=1 .^{1}$ We refer to this formulation as the unrestricted mean-variance (MeVU) portfolio.

When considering the case where the investor risk aversion tends to the infinity $(\gamma \rightarrow \infty)$ the problem can be represented as

$$
\begin{gathered}
\min _{w_{t}} w_{t}^{\top} \Sigma_{t} w_{t}, \\
\text { s.t. } w_{t}^{\top} e=1 .
\end{gathered}
$$

In this case, the investor only cares about reducing the portfolio risk, without considering the expected return. This is an important portfolio policy since expected returns is subjected to more estimation errors when compared to the estimation of covariances (Merton, 1980). We refer to it as the unrestricted minimum variance portfolio (MiVU).

One popular variation of the traditional mean-variance formation is to include a constraint on short-sales. Jagannathan \& Ma (2003) show that including this restriction alleviates estimation error in portfolio weights. The constrained-version formulation for the mean-variance problem can be specified as

$$
\begin{gathered}
\min _{w_{t}} w_{t}^{\top} \Sigma_{t} w_{t}-\frac{1}{\gamma} w_{t}^{\top} \mu_{t}, \\
\text { s.t. } w_{t}^{\top} e=1 \\
w_{t} \geq 0
\end{gathered}
$$

where the restriction $w_{t} \geq 0$ represents the short-sale restriction. We refer to it as the restricted mean-

\footnotetext{
${ }^{1}$ In unreported results, we also have considered the performance of mean-variance policies under alternative levels of the risk aversion coefficient. The results are very similar to those reported here and are promptly available upon request.
} 
variance (MeVC) portfolio . Similarly, the restricted minimum variance (MiVC) portfolio is given by:

$$
\begin{gathered}
\min _{w_{t}} w_{t}^{\top} \Sigma_{t} w_{t}, \\
\text { s.t. } w_{t}^{\top} e=1 \\
w_{t} \geq 0 .
\end{gathered}
$$

Norm-constrained mean-variance. We follow DeMiguel et al. (2014) and consider a class of mean-variance portfolios with norm restriction (NCMV) given by:

$$
\begin{gathered}
\min _{w_{t}} w_{t}^{\top} \Sigma_{t} w_{t}-\frac{1}{\gamma} w_{t}^{\top} \mu_{t}, \\
\text { s.t. } w_{t}^{\top} e=1 \\
\left\|w_{t}-w_{0 t}\right\|_{1}=\sum_{i=1}^{N}\left|\left(w_{t}\right)_{i}-\left(w_{0 t}\right)_{i}\right| \leq \delta
\end{gathered}
$$

where $w_{0 t}$ is the vector of weights of the MeVC portfolio and $\delta$ is the maximum deviation from the norm. The norm restriction requires that the weights of this portfolio remain close to the weights of the MeVC portfolio, as the aggregate absolute distance between the weights cannot be greater than the norm. We follow DeMiguel et al. (2014) and use the 1-norm in relation to the restricted mean-variance. Moreover, we consider the maximum deviation to the norm, $\delta$, to be equal to $2.5 \%, 5.0 \%$ and $10.0 \%$. We refer to these portfolios as NCMV2.5, NCMV5, and NCMV10, respectively.

Volatility timing and reward-to-risk. We implement the volatility timing (VT) portfolio policy proposed in Kirby \& Ostdiek (2012). These policies greatly simplify the computation of optimal weights by setting offdiagonal covariance elements equal to 0 and therefore reducing estimation error. The VT policy assumes that portfolio weight in the $i^{\text {th }}$ asset is inversely proportional to its estimated variance, i.e.,

$$
\hat{w}_{i t}=\frac{\left(\frac{1}{\hat{\sigma}_{i t}^{2}}\right)^{\eta}}{\sum_{i=1}^{N}\left(\frac{1}{\hat{\sigma}_{i t}^{2}}\right)^{\eta}}
$$

where $\hat{\sigma}_{i t}$ is the volatility of the $i^{t h}$ asset. The parameter $\eta$ measures the aggressiveness of the allocation. The tuning parameter $\eta \geq 0$ determines how aggressively we adjust the portfolio weights in response to changes in the assets' variances. As $\eta \rightarrow 0$ we recover the equally-weighted portfolios, and as $\eta \rightarrow \infty$ the weight on the asset with lowest variance approaches 1 . Thus, large values of $\eta$, can shrink the portfolio weights towards the less risky assets. We follow Kirby \& Ostdiek (2012) and considered $\eta$ equals to 1,2 and 4 thus yielding the VT1, VT2, and VT4 policies, respectively.

Kirby \& Ostdiek (2012) also put forward a variation of the VT policy in order to take into account 
information about conditional expected returns, and therefore selecting assets according to their riskreturn trade-off. The reward-to-risk $(\mathrm{RwR})$ policy is defined as

$$
\hat{w}_{i t}=\frac{\left(\frac{\hat{\mu}_{i t}^{+}}{\hat{\sigma}_{i t}^{2}}\right)^{\eta}}{\sum_{i=1}^{N}\left(\frac{\hat{\mu}_{i t}^{+}}{\hat{\sigma}_{i t}^{2}}\right)^{\eta}}
$$

where $\hat{\mu}_{i t}^{+}=\max \left(\hat{\mu}_{i t}, 0\right)$ and $\hat{\mu}_{i t}$ is the $i^{t h}$ asset expected return i.e. $\mu_{i t}=\frac{1}{T} \sum_{i=1}^{T} R_{i}$ where $T$ is the length of the estimation window. We follow Kirby \& Ostdiek (2012) and implement the RwR polcy by considered $\eta$ equals to 1,2 and 4 thus yielding the RwR1, RwR2, and RwR4 policies, respectively.

Finally, we also consider a variation of the RwR policy that aims at improving the estimation of expected returns by considering a factor model. One immediate choice is to assume that that a conditional version of the capital asset pricing. The conditional CAPM implies that the cross-sectional variation in conditional expected excess returns is due to cross-sectional variation in conditional betas. This approach, however, can also be extended to multiple risk factors. The resulting weights for the RwR strategy is

$$
\hat{w}_{i t}=\frac{\left(\frac{\bar{\beta}_{i t}^{+}}{\hat{\sigma}_{i t}^{2}}\right)^{\eta}}{\sum_{i=1}^{N}\left(\frac{\bar{\beta}_{i t}^{+}}{\hat{\sigma}_{i t}^{2}}\right)^{\eta}}
$$

where $\bar{\beta}_{i t}^{+}=\max \left(\bar{\beta}_{i t}, 0\right)$ and $\bar{\beta}_{i t}=(1 / K) \sum_{j=1}^{K} \beta_{i j, t}$ is the average conditional beta of asset $i$ with respect to the $K$ factors. To implement this policy, we consider the 4-factor model proposed by Carhart (1997) as an extension to the 3-factor model proposed by Fama \& French (1992). Similar as in the VT policies, we considered $\eta$ equals to 1, 2 and 4 thus yielding the RwR4F1, RwR4F2, and RwR4F4 policies, respectively.

\section{Covariance models}

In this Section we describe the alternative approaches we use to model covariances in our empirical exercise. We initially describe the static models based of the shrinkage methods proposed in Ledoit \& Wolf (2003a,b, 2004). Later, we describe the dynamic specifications based on the multivariate GARCH family.

\subsection{Shrinkage methods}

The shrinkage method used to model covariances is based on the bias-variance trade-off. The method considers that a way to obtain a better covariance estimator is to simply take a weighted average between a biased estimator, with little estimation error, and an unbiased one, but with a lot of estimation error. This process is refereed to as the shrinkage of an unbiased estimator towards a target, represented by the biased estimator. 
This idea can be summarized as

$$
\Sigma_{t}=\hat{\delta} F_{t}+(1-\hat{\delta}) S_{t}
$$

where $0 \leq \hat{\delta} \leq 1$ is the estimated shrinkage intensity, $F$ is usually a highly structured covariance matrix, and $S$ is an unstructured estimator. The sample covariance matrix estimated as $\hat{S}_{t}=(1 / T) \sum_{t=1}^{T}\left(R_{t}-\hat{\mu}_{t}\right)\left(R_{t}-\hat{\mu}_{t}\right)^{\prime}$ is most frequently used as this unstructured estimator. The structured estimator usually reflects important characteristics of the variable being estimated. We considered as target covariances those considered in Ledoit $\&$ Wolf (2003a,b, 2004).

Ledoit \& Wolf (2003a) uses a model of constant correlation between the assets in order to define the target structured estimator $F$. To define this covariance matrix, let $S_{t}$ be the sample covariance and $s_{i j, t}$ be the element of $S_{t}$ at row $i$ and column $j$. So the sample correlation is defined as $r_{i j, t}=s_{i j, t} / \sqrt{s_{i i, t} s_{j j, t}}$, whose average is $\bar{r}_{t}=\frac{2}{(N-1) N} \sum_{i=1}^{N-1} \sum_{j=i+1}^{N} r_{i j, t}$. Therefore, the constant correlation matrix $F_{t}$ can be obtained using the sample variances and the sample average correlation, i.e. $f_{i i, t}=s_{i i, t}$ and $f_{i j, t}=\bar{r}_{t} \sqrt{s_{i i, t} s_{j j, t}}$, where $f_{i j, t}$ be the element of $F_{t}$ at row $i$ and column $j$.

Ledoit \& Wolf (2003b) assume an one factor market model for the return of asset $j$ at time $t$ :

$$
r_{j, t}=\alpha_{j}+\beta_{j} r_{M, t}+\epsilon_{j, t}
$$

where $r_{M, t}$ is the market index return at time $t, \beta_{j}$ is the load on the market factor, $\alpha_{j}$ is the intercept and $\epsilon_{j, t}$ is the error. Assuming that $r_{M, t}$ and $\epsilon_{j, t}$ are uncorrelated and that $\epsilon_{i, t}$ and $\epsilon_{j, t}$ are uncorrelated for $i \neq j$, the structured target covariance matrix is defined as

$$
F_{t}=s_{m, t} B B^{\top}+D_{t}
$$

where $B$ is the vector of $\beta \mathrm{s}, s_{m, t}$ is the sample variance of $r_{M, t}$ and $D_{t}$ in the diagonal matrix consisting of the variance of the sample errors. Finally, Ledoit \& Wolf (2004) use the identity matrix as the target structured estimator, i.e. $F_{t}=\mathbf{I}$.

One appealing aspect of the three shrinkage methods described above is that the computation of the shrinkage intensity $\hat{\delta}$ is based on closed form expressions. A description of these expressions can be found in Ledoit \& Wolf $(2003 \mathrm{a}, \mathrm{b}, 2004)$.

\subsection{Multivariate GARCH models}

The conditional covariance models considered in this paper belong to the class of multivariate GARCH models. For that purpose, we assume that the multivariate system of asset returns is conditionally heteroskedastic and follows $R_{t}=z_{t}\left(\Sigma_{t}\right)^{1 / 2}$. To model $\Sigma_{t}$, we implement 8 specifications commonly used in portfolio selection problems such as those employed in Engle \& Sheppard (2008) and Becker et al. (2014). Next we briefly describe 
each of the conditional covariance specification employed in the paper.

Exponentially weighted moving average (EWMA): The EWMA model is defined as

$$
\Sigma_{t}=\alpha R_{t-1}{ }^{\prime} R_{t-1}+(1-\alpha) \Sigma_{t-1}
$$

where $\alpha$ is a non-negative parameter. When the $\alpha$ is set to a fixed value of 0.04 , the EWMA is equivalent to the popular Riskmetrics approach. Zaffaroni (2008) shows that although it permits sizable computational gains and provide a simple way to impose positive semi-definiteness of the resulting conditional covariance matrices, the Riskmetrics delivers non-consistent estimates. Therefore, in our implementation of the EWMA specification the parameter $\alpha$ is estimated via maximum likelihood; see details below.

Optimal rolling estimator (ORE): The general rolling estimator is defined as $\Sigma_{t}=\sum_{k=1}^{\infty} \Omega_{t-k} \odot R_{t-k}{ }^{\prime} R_{t-k}$, where $\Omega_{t-k}$ is a symmetric matrix of weights and $\odot$ denotes the element-by-element multiplication. This structure admits a wide range of potential weighting schemes. Foster \& Nelson (1996) show that the optimal strategy is to let the weights decline in an exponential fashion as the magnitude of $k$ increases. Their procedure, however, implies a different decay rate for each element of the conditional covariance matrix $\Sigma_{t}$. Because this makes it difficult to ensure that the resulting matrix is positive definite, we follow Fleming et al. (2001, 2003) and impose the restriction $\Omega_{i j, t}=\Omega_{t}$ for all $i$ and $j$. In this case, the optimal weighting scheme is given by $\Omega_{t-k}=\alpha \exp (-\alpha k) \iota^{\prime} \iota$ where $\alpha$ is the decay rate and $\iota$ is a vector of ones. Therefore, the rolling estimator can be rewritten as

$$
\Sigma_{t}=\alpha \exp (-\alpha) R_{t-1}{ }^{\prime} R_{t-1}+\exp (-\alpha) H_{t-1}
$$

where $\alpha$ is a nonnegative parameter which is estimated via maximum likelihood; see details below. In (13) a single parameter $(\alpha)$ controls the rate at which the weights decay with the lag length. This parsimony facilitates estimation specially when the dimension is high. The ORE specification has been applied in many portfolio selection problems such as in Fleming et al. (2001, 2003) and Pooter et al. (2008). Fleming et al. (2003), in particular, point out that covariance matrix forecasts based on the ORE specification results in better portfolios in comparison to those obtained with other (unrestricted) multivariate GARCH models. The authors argue that the smoothness of the rolling estimator as the main reason for this.

Scalar VECH: The scalar VECH specification of Bollerslev et al. (1988) is defined as

$$
\Sigma_{t}=C^{\prime} C+\alpha R_{t-1} R_{t-1}{ }^{\prime}+\beta H_{t-1}
$$

Instead of estimating $N(N+1) / 2$ unique elements of $C$, we employ the variance targeting technique as suggested 
in Engle \& Mezrich (1996). The general idea is to estimate the intercept matrix by an auxiliary estimator that is given by $\hat{C}^{\prime} \hat{C}=\bar{S}(1-\alpha-\beta)$, where $\bar{S}=\frac{1}{T} \sum_{t=1}^{T} R_{t} R_{t}{ }^{\prime}$, thus yielding the variance-targeting scalar VECH model

$$
\Sigma_{t}=\bar{S}(1-\alpha-\beta)+\alpha R_{t-1} R_{t-1}^{\prime}+\beta H_{t-1}
$$

which is covariance-stationary provided that $\alpha+\beta<1$.

Orthogonal GARCH (O-GARCH): The O-GARCH model of Alexander (2001) belongs to a class of factor models and is able to achieve significant computational gains via dimensionality reduction. The O-GARCH model is given by $\Sigma_{t}=W \Omega_{t} W$, where $W$ is a $N \times k$ matrix whose columns are given by the first $k$ eigenvectors of the $t \times N$ matrix of asset returns, and $\Omega_{t}$ is a $k \times k$ diagonal matrix whose elements are given by $h_{f_{k t}}$ where $h_{f_{k t}}$ is the conditional variance of the $k$-th principal component and follows a $\operatorname{GARCH}(1,1)$ process. We follow Engle \& Sheppard (2008) and implement the O-GARCH model using 3 principal components.

Conditional correlation models: This class of models is defined as $H_{t}=D_{t} \Psi_{t} D_{t}$, where $D_{t}$ is a $N \times N$ diagonal matrix with diagonal elements given by $h_{i, t}$, where $h_{i, t}$ is the conditional variance of the $i$-th asset and follows a $\operatorname{GARCH}(1,1)$ process, and $\Psi_{t}$ is a symmetric conditional correlation matrix with elements $\rho_{i j, t}$, where $\rho_{i i, t}=1, i, j=1, \ldots, N$. We consider 4 alternative specifications to model $\Psi_{t}$ : (i) the constant conditional correlation (CCC) model of Bollerslev (1990), (ii) the dynamic conditional correlation (DCC) model of Engle (2002), (iii) the asymmetric DCC (ASYDCC) of Cappiello et al. (2006), (iv) the dynamic equicorrelation (DECO) model of Engle \& Kelly (2012). Engle \& Colacito (2006) and Engle \& Sheppard (2008) study the performance of alternative conditional correlation models in portfolio selection problems.

Multivariate GARCH models are typically estimated via quasi maximum likelihood (QML). However, this estimator is found to be severely biased in large dimensions; see, for instance, Engle et al. (2008) and Hafner \& Reznikova (2012). In this paper, the parameters of the EWMA, ORE, and VECH specifications are estimated with the composite likelihood (CL) method proposed by Engle et al. (2008). As for the conditional correlation models, their estimation can be conveniently divided into volatility part and correlation part. The volatility part refers to estimating the univariate conditional variances which is done by QML assuming Gaussian innovations. The parameters of the correlation matrix in the DCC and ASYDCC models are estimated using the CL method. As pointed out by Engle et al. (2008), the CL estimator provides more accurate parameter estimates in comparison to the two-step procedure proposed by Engle (2002), especially in large problems. 


\section{Methodology for evaluating portfolio performance}

To implement the portfolio policies described in Section 2 alongside the covariance models discussed in Section 3, we adopt a recursive estimation approach that works as follows. First, we use an initial estimation window of $T=1500$ daily observations to estimate all covariance models (11 models). As for the dynamic ones, we also obtain one-step-ahead forecasts of the conditional covariance matrix for the period 1501. Second, we implement each of the portfolio policies (16 in total) using each of the estimated covariances, therefore yielding a total of $16 \times 11=176$ different portfolios. Third, we compute portfolio statistics for each for period 1501 using the methodology described in Section 4. Fourth, we add one observation to the estimation window and repeat this process until the end of the data set is reached. We end up with a sample of $L-T$ pseudo out-of-sample observations, where $L$ is the length of the data set, which are used to evaluate the performance of each of the 176 portfolios. Portfolos are re-balanced on a daily basis.

Holding the portfolio $w_{t}$ for one day gives the out-of-sample portfolio return at time $t+1: R_{p, t}=w_{t}^{\prime} R_{t+1}$ where $R_{t+1}$ is the vector of asset returns. We use the time series of out-of-sample portfolio returns and weights of each portfolio policy described in Section 2 to evaluate the performance in terms of average return $(\hat{\mu})$ and standard deviation (volatility) of returns $(\widehat{\sigma})$. These statistics are calculated as follows:

$$
\begin{aligned}
& \hat{\mu}=\frac{1}{T-1} \sum_{t=1}^{T-1} w_{t}^{\prime} R_{t+1} \\
& \hat{\sigma}=\sqrt{\frac{1}{T-1} \sum_{t=1}^{T-1}\left(w_{t}^{\prime} R_{t+1}-R_{p}\right)^{2} .}
\end{aligned}
$$

where $R_{p}$ is the realized portfolio average return.

A crucial aspect that must be taken into account is the impact of transaction costs on the performance of optimal portfolios (Han, 2006). As Kolm et al. (2014) points out, transaction costs consist of direct costs, such as commissions and taxes, bid-ask spread, and indirect costs, such as slippage. As in Olivares-Nadal \& DeMiguel (2015), for small trades, which do not impact the market price, the transaction cost is assumed to proportional to the amount traded on each asset. To take into account the impact of proportional transaction costs, we follow Della Corte et al. (2008) and Thornton \& Valente (2012) and compute the portfolio return net of transaction costs $\left(R_{p, t}^{\text {net }}\right)$. This calculation is performed as

$$
R_{p, t}^{\text {net }}=\left(1-c \cdot \text { turnover }_{t}\right)\left(1+w_{t}^{\prime} R_{t+1}\right)-1
$$

where $c$ is the fee that must be paid for each transaction and turnover $t_{t}$ is the portfolio turnover at time $t$, defined as the fraction of wealth traded between periods $t$ and $t+1$, i.e

$$
\text { turnover }_{t}=\sum_{j=1}^{N}\left(\left|w_{j, t+1}-w_{j, t}\right|\right) \text {. }
$$


To compute (15), it is necessary to set an appropriate value for the transaction cost $c$. French (2008) estimates how much Americans spend on transaction costs each year for different active investing options, and compare it with the cost of passive investing. He finds that Americans spend 21 basis points (bp) in total trading, as a fraction of the total portfolio. Therefore, in order to evaluate the impact of transaction costs on the performance of the alternative portfolio selection policies, we follow Kirby \& Ostdiek (2012) and consider three alternative scenarios of transaction costs: 0 bp (no transaction costs), 20 bp (intermediate level of transaction costs), and $50 \mathrm{bp}$ (high level of transaction costs). ${ }^{2}$

Upon computing the average and the standard deviation of the portfolio return net of transaction costs for based on each of the three levels of transaction costs considered in the paper, we compute the average portfolio turnover over all observations as well as the risk-adjusted portfolio return net of transaction costs measured by the Sharpe ratio (SR), which is defined as

$$
S R=\frac{\bar{R}_{p}^{\text {net }}}{\sigma^{\text {net }}}
$$

where ${\overline{R_{p}}}^{\text {net }}$ and $\sigma^{\text {net }}$ are, respectively, the average and the standard deviation of portfolio returns net of transaction costs.

In order to assess the relative performance of portfolios obtained with dynamic covariance models with respect to those obtained with static models, we implement the bootstrap test for the differences in SR proposed by Ledoit \& Wolf (2008). For that purpose, we select as main benchmark the portfolios obtained with the shrinkage method proposed in Ledoit \& Wolf (2004). In other words, we test for the differences in Sharpe ratios obtained with a dynamic covariance model with respect to those obtained with a static model within the same portfolio selection policy.

Finally, we also follow Fleming et al. (2001) and Kirby \& Ostdiek (2012) and use a utility-based approach to measure the value of the performance gains associated with employing a given portfolio strategy along with a specific choice for the covariance model. We assume the investor has a quadratic utility function given by:

$$
U\left(R_{p, t}\right)=W_{0}\left(1+R_{p, t}-\frac{\gamma}{2(1+\gamma)}\left(1+R_{p, t}\right)^{2}\right)
$$

where $R_{p, t}=w_{t-1}^{\prime} R_{t}$ is the portfolio return, $\gamma$ is the investor's relative risk aversion and $W_{0}$ is the initial wealth. In order to compare two alternative portfolio strategies $\left(R_{p 1}\right.$ and $\left.R_{p 2}\right)$, we determine the maximum performance fee a risk-averse investor would be willing to pay to switch from using one portfolio policy to another. That is, we determine the value of $\Delta$ such that

$$
\sum_{t=1}^{T-1} U\left(R_{p 1, t}\right)=\sum_{t=1}^{T-1} U\left(R_{p 2, t}-\Delta\right)
$$

This constant represents the maximum return the investor would be willing to sacrifice each period in order

\footnotetext{
${ }^{2}$ Kirby \& Ostdiek (2012) point out that "transaction costs might be less of an issue for large institutional investors. Establishing or liquidating a portfolio position could plausibly cost as little as 5 bp for such investors."
} 
to capture the performance gains associated with switching to the second portfolio policy/covariance model. We report the value of $\Delta$ as an annualized basis point fee for an investor with risk aversion coefficient $\gamma=1 .^{3}$ Moreover, we compute the value of $\Delta$ taking the portfolio policies obtained with the PARA static covariance specification of Ledoit \& Wolf (2004) as a benchmark.

\section{Empirical evaluation}

\subsection{Data}

Our empirical exercise is based on a sample composed of 50 most traded stocks belonging to the S\&P100 index between $01 / 01 / 2004$ and $31 / 12 / 2013$. The sample goes through periods of growth, recession and economic recovery, including periods of high and low market volatility, so that we do not restrict the results to a specific sample characteristic. The sample contains 2516 observations with daily data of returns, computed as the logarithmic difference of the closing prices. We report in Table 12 descriptive statistics for the sample used in the paper.

\subsection{Results}

The presentation of the results is organized in five parts to facilitate the analysis and discussion. Initially, we discuss the performance of each portfolio strategy in terms of gross returns. Second, we present the results in terms of portfolio risk measured by the standard deviation of returns. Third, we report the results for the portfolio turnover. Fourth, we discuss the results in terms of risk-adjusted portfolio returns measured by the SR for alternative levels of transaction costs. Finally, we report the annualized performance fee that a risk-averse investor with quadratic utility adopting a given portfolio policy is willing to pay in order to use the PARA static covariance specification of Ledoit \& Wolf (2004). In each part, we report the results for the portfolio policies obtained with dynamic covariance models as well as those obtained with static covariance models. To facilitate the exposition of results, we refer to each portfolio policy and each covariance model using the acronyms detailed in Tables 10 and 11 in the Appendix, respectively.

Portfolio gross returns. Table 1 shows the average gross returns (i.e. before transaction costs) obtained by each portfolio policy and the corresponding covariance model used. In the vast majority of instances, average gross return obtained using dynamic specifications is equal or superior to those obtained with static specifications. These differences are more substantial for the group of mean-variance portfolio policies. Overall, the best results in terms are obtained with the RwRs4 policy with the EWMA and ORE specifications $(0.11 \%$ and $0,10 \%$, respectively).

\footnotetext{
${ }^{3}$ In unreported results, we also computed the annualized performance fee $\delta$ assuming an investor with risk aversion coefficient $\gamma=\{2,5,10\}$. The results are qualitatively similar to those reported here and are promptly available upon request.
} 


\section{Table 1: Portfolio gross returns}

The table reports the daily average gross return (i.e. before transaction costs) obtained by the each portfolio policy alongside each covariance model over the out-of-sample period. Figures are reported in percentages. The portfolio policies are described in Section 2 whereas the covariance models are described in Section 3. The description of each acronym for the portfolio polices and for the covariance models are reported in Tables 10 and 11 .

\begin{tabular}{|c|c|c|c|c|c|c|c|c|c|c|c|c|c|c|c|c|c|}
\hline Strategy & MiVU & $\mathrm{MeVU}$ & MiVC & $\mathrm{MeVC}$ & NCMV2.5 & NCMV5 & NCMV10 & VT1 & VT2 & VT4 & RwRS1 & RwRS2 & RwRS4 & RwR4F1 & RwR4F2 & RwR4F4 & Average \\
\hline \multicolumn{18}{|c|}{ Dynamic covariance models } \\
\hline EWMA & 0.071 & 0.076 & 0.070 & 0.071 & 0.071 & 0.072 & 0.074 & 0.062 & 0.063 & 0.064 & 0.066 & 0.081 & 0.108 & 0.059 & 0.060 & 0.062 & 0.071 \\
\hline ORE & 0.064 & 0.065 & 0.060 & 0.061 & 0.061 & 0.062 & 0.064 & 0.062 & 0.061 & 0.057 & 0.064 & 0.077 & 0.102 & 0.059 & 0.058 & 0.059 & 0.065 \\
\hline VECH & 0.077 & 0.083 & 0.072 & 0.074 & 0.073 & 0.074 & 0.075 & 0.062 & 0.063 & 0.062 & 0.064 & 0.076 & 0.099 & 0.060 & 0.062 & 0.066 & 0.071 \\
\hline OGARCH & 0.065 & 0.064 & 0.056 & 0.056 & 0.057 & 0.058 & 0.058 & 0.061 & 0.059 & 0.053 & 0.060 & 0.064 & 0.076 & 0.057 & 0.057 & 0.059 & 0.060 \\
\hline $\mathrm{CCC}$ & 0.060 & 0.060 & 0.062 & 0.062 & 0.063 & 0.063 & 0.063 & 0.062 & 0.062 & 0.062 & 0.061 & 0.066 & 0.077 & 0.059 & 0.059 & 0.062 & 0.063 \\
\hline DCC & 0.049 & 0.105 & 0.074 & 0.075 & 0.075 & 0.076 & 0.077 & 0.062 & 0.062 & 0.062 & 0.061 & 0.066 & 0.077 & 0.059 & 0.059 & 0.062 & 0.069 \\
\hline DECO & 0.069 & 0.069 & 0.060 & 0.060 & 0.060 & 0.060 & 0.059 & 0.062 & 0.062 & 0.062 & 0.061 & 0.066 & 0.077 & 0.059 & 0.059 & 0.062 & 0.063 \\
\hline ASYDCC & 0.077 & 0.078 & 0.073 & 0.075 & 0.074 & 0.075 & 0.077 & 0.061 & 0.062 & 0.062 & 0.062 & 0.075 & 0.104 & 0.057 & 0.057 & 0.058 & 0.070 \\
\hline Average & 0.066 & 0.075 & 0.066 & 0.067 & 0.067 & 0.067 & 0.068 & 0.062 & 0.061 & 0.061 & 0.063 & 0.072 & 0.090 & 0.059 & 0.059 & 0.061 & 0.066 \\
\hline \multicolumn{18}{|c|}{ Static covariance models } \\
\hline CORR & 0.052 & 0.052 & 0.055 & 0.055 & 0.056 & 0.056 & 0.057 & 0.061 & 0.058 & 0.052 & 0.061 & 0.066 & 0.081 & 0.057 & 0.055 & 0.054 & 0.058 \\
\hline PARA & 053 & 0.052 & 0.055 & 0.055 & 0.056 & 0.0 & 0.058 & 0.061 & 0.059 & 0.053 & 0.06 & 0.067 & 0.0 & 0.057 & 0.056 & 0.054 & 0.059 \\
\hline MARKET & 0.052 & 0.052 & 0.055 & 0.055 & 0.056 & 0.057 & 0.058 & 0.061 & 0.058 & 0.052 & 0.061 & 0.066 & 0.081 & 0.057 & 0.055 & 0.054 & 0.058 \\
\hline Average & 0.052 & 0.052 & 0.055 & 0.055 & 0.056 & 0.057 & 0.058 & 0.061 & 0.058 & 0.053 & 0.061 & 0.067 & 0.081 & 0.057 & 0.055 & 0.054 & 0.058 \\
\hline
\end{tabular}

Portfolio standard deviation. Table 2 reports the standard deviation of the gross portfolio returns for each portfolio policy. The comparative results among dynamic and static covariance specifications are rather mixed according to this performance measure. We observe that the results are similar among policies when using dynamic covariances or static covariances. An exception is the DCC specification which delivered portfolios with much higher standard deviations in comparison to other dynamic or static counterparts.

Table 2: Standard deviation of portfolio returns

The table reports the standard deviation of portfolio gross returns obtained by the each portfolio policy alongside each covariance model over the out-of-sample period. Figures are reported in percentages. The portfolio policies are described in Section 2 whereas the covariance models are described in Section 3. The description of each acronym for the portfolio polices and for the covariance models are reported in Tables 10 and 11

\begin{tabular}{|c|c|c|c|c|c|c|c|c|c|c|c|c|c|c|c|c|c|}
\hline Strategy & MiVU & $\mathrm{MeVU}$ & MiVC & $\mathrm{MeVC}$ & NCMV2.5 & NCMV5 & NCMV10 & VT1 & VT2 & VT4 & RwRS1 & RwRS2 & RwRS4 & RwR4F1 & RwR4F2 & RwR4F4 & Average \\
\hline \multicolumn{18}{|c|}{ Dynamic covariance models } \\
\hline EWMA & 0.687 & 0.708 & 0.692 & 0.694 & 0.683 & 0.676 & 0.664 & 0.899 & 0.778 & 0.708 & 0.904 & 0.887 & 1.021 & 0.966 & 0.932 & 0.918 & 0.801 \\
\hline ORE & 0.648 & 0.654 & 0.700 & 0.701 & 0.692 & 0.685 & 0.675 & 0.899 & 0.780 & 0.715 & 0.910 & 0.902 & 1.061 & 0.969 & 0.934 & 0.909 & 0.802 \\
\hline VECH & 0.726 & 0.751 & 0.696 & 0.697 & 0.687 & 0.679 & 0.668 & 0.901 & 0.779 & 0.713 & 0.902 & 0.871 & 0.971 & 0.969 & 0.936 & 0.926 & 0.804 \\
\hline OGARCH & 0.690 & 0.692 & 0.723 & 0.723 & 0.715 & 0.707 & 0.694 & 0.928 & 0.810 & 0.732 & 0.939 & 0.898 & 0.915 & 1.005 & 0.988 & 0.965 & 0.820 \\
\hline $\mathrm{CCC}$ & 0.675 & 0.675 & 0.711 & 0.711 & 0.702 & 0.695 & 0.685 & 0.913 & 0.793 & 0.723 & 0.910 & 0.869 & 0.937 & 0.979 & 0.949 & 0.946 & 0.804 \\
\hline DCC & 1.237 & 2.936 & 0.741 & 0.742 & 0.733 & 0.726 & 0.716 & 0.913 & 0.793 & 0.723 & 0.910 & 0.869 & 0.937 & 0.979 & 0.949 & 0.946 & 0.991 \\
\hline DECO & 0.707 & 0.710 & 0.713 & 0.712 & 0.706 & 0.699 & 0.688 & 0.913 & 0.793 & 0.723 & 0.910 & 0.869 & 0.937 & 0.979 & 0.949 & 0.946 & 0.810 \\
\hline ASYDCC & 0.682 & 0.706 & 0.725 & 0.732 & 0.717 & 0.710 & 0.698 & 1.003 & 0.913 & 0.793 & 0.991 & 1.023 & 1.322 & 1.056 & 1.064 & 1.083 & 0.889 \\
\hline Average & 0.757 & 0.979 & 0.713 & 0.714 & 0.704 & 0.697 & 0.686 & 0.921 & 0.805 & 0.729 & 0.922 & 0.898 & 1.013 & 0.988 & 0.963 & 0.955 & 0.840 \\
\hline \multicolumn{18}{|c|}{ Static covariance models } \\
\hline CORR & 0.666 & 0.667 & 0.716 & 0.716 & 0.708 & 0.702 & 0.691 & 0.921 & 0.803 & 0.732 & 0.928 & 0.887 & 0.931 & 0.999 & 0.978 & 0.953 & 0.812 \\
\hline PARA & 0.669 & 0.670 & 0.718 & 0.718 & 0.709 & 0.702 & 0.692 & 0.925 & 0.809 & 0.733 & 0.932 & 0.895 & 0.950 & 1.003 & 0.984 & 0.962 & 0.817 \\
\hline MARKET & 0.668 & 0.669 & 0.717 & 0.717 & 0.709 & 0.702 & 0.692 & 0.921 & 0.803 & 0.732 & 0.928 & 0.887 & 0.931 & 0.999 & 0.978 & 0.953 & 0.813 \\
\hline Average & 0.668 & 0.669 & 0.717 & 0.717 & 0.709 & 0.702 & 0.692 & 0.922 & 0.805 & 0.732 & 0.929 & 0.890 & 0.938 & 1.001 & 0.980 & 0.956 & 0.814 \\
\hline
\end{tabular}

Turnover. The turnover obtained for each portfolio policy is reported in Table 3. The most striking result is that all portfolios obtained with dynamic covariance models displayed much higher turnover in comparison to those obtained with their static counterparts. For instance, when obtaining portfolios according to the MeVU policy, dynamic covariance obtained an average turnover of 0.77 , whereas the same figure for the static co- 
variance models is 0.022 . We also observe that i) constrained mean-variance policies displayed lower turnovers with respect to unconstrained policies, and ii) policies that ignore off-diagonal covariance elements, such as VT and $\mathrm{RwR}$ policies, also achieved lower turnover with respect all mean-variance policies. It is also interesting to associate the results in Table 3 with those reported in table 1. In general, we observe that the higher portfolio average returns obtained with dynamic covariance specifications is usually associated with a higher portfolio turnover. The most extreme case is that of the DCC specification that has a reasonably superior return (0.069 on average), when compared to the other portfolios, but it was coupled with the highest turnover among all portfolios observed (0.991 on average). Therefore, even though dynamic specifications with greater structure are linked to higher average returns, they come associated with much higher portfolio instability.

\section{Table 3: Portfolio turnover}

The table reports the portfolio turnover obtained by the each portfolio policy alongside each covariance model over the outof-sample period. Figures are reported in percentages. The computation of turnovers is described in Section 4 . The portfolio policies are described in Section 2 whereas the covariance models are described in Section 3. The description of each acronym for the portfolio polices and for the covariance models are reported in Tables 10 and 11.

\begin{tabular}{|c|c|c|c|c|c|c|c|c|c|c|c|c|c|c|c|c|c|}
\hline Strategy & MiVU & $\mathrm{MeVU}$ & MiVC & $\mathrm{MeVC}$ & NCMV2.5 & NCMV5 & NCMV10 & VT1 & VT2 & VT4 & RwRS1 & RwRS2 & RwRS4 & RwR4F1 & RwR4F2 & RwR4F4 & Average \\
\hline \multicolumn{18}{|c|}{ Dynamic covariance models } \\
\hline EWMA & 0.296 & 0.307 & 0.074 & 0.074 & 0.077 & 0.079 & 0.083 & 0.016 & 0.027 & 0.046 & 0.021 & 0.031 & 0.042 & 0.017 & 0.028 & 0.046 & 0.079 \\
\hline ORE & 0.089 & 0.090 & 0.023 & 0.023 & 0.024 & 0.025 & 0.027 & 0.008 & 0.010 & 0.014 & 0.016 & 0.019 & 0.021 & 0.011 & 0.013 & 0.018 & 0.027 \\
\hline VECH & 0.473 & 0.496 & 0.141 & 0.139 & 0.145 & 0.149 & 0.158 & 0.030 & 0.055 & 0.095 & 0.032 & 0.055 & 0.082 & 0.030 & 0.054 & 0.093 & 0.139 \\
\hline OGARCH & 0.051 & 0.052 & 0.031 & 0.032 & 0.032 & 0.034 & 0.037 & 0.011 & 0.014 & 0.014 & 0.018 & 0.025 & 0.035 & 0.013 & 0.019 & 0.032 & 0.028 \\
\hline $\mathrm{CCC}$ & 0.403 & 0.393 & 0.216 & 0.216 & 0.222 & 0.228 & 0.238 & 0.055 & 0.112 & 0.206 & 0.056 & 0.108 & 0.185 & 0.053 & 0.100 & 0.180 & 0.186 \\
\hline DCC & 1.828 & 4.156 & 0.300 & 0.296 & 0.307 & 0.314 & 0.329 & 0.055 & 0.112 & 0.206 & 0.056 & 0.108 & 0.185 & 0.053 & 0.100 & 0.180 & 0.537 \\
\hline DECO & 0.264 & 0.261 & 0.209 & 0.210 & 0.215 & 0.221 & 0.227 & 0.055 & 0.112 & 0.206 & 0.056 & 0.108 & 0.185 & 0.053 & 0.100 & 0.180 & 0.166 \\
\hline ASYDCC & 0.410 & 0.404 & 0.170 & 0.163 & 0.174 & 0.178 & 0.186 & 0.029 & 0.055 & 0.112 & 0.031 & 0.052 & 0.059 & 0.028 & 0.051 & 0.093 & 0.137 \\
\hline Average & 0.477 & 0.770 & 0.146 & 0.144 & 0.150 & 0.154 & 0.161 & 0.032 & 0.062 & 0.112 & 0.036 & 0.063 & 0.099 & 0.032 & 0.058 & 0.103 & 0.162 \\
\hline \multicolumn{18}{|c|}{ Static covariance models } \\
\hline CORR & 0.019 & 0.021 & 0.005 & 0.005 & 0.005 & 0.005 & 0.006 & 0.007 & 0.006 & 0.005 & 0.016 & 0.019 & 0.023 & 0.010 & 0.011 & 0.012 & 0.011 \\
\hline PARA & 0.021 & 0.022 & 0.005 & 0.005 & 0.005 & 0.006 & 0.006 & 0.007 & 0.006 & 0.005 & 0.016 & 0.019 & 0.022 & 0.010 & 0.011 & 0.012 & 0.011 \\
\hline MARKET & 0.021 & 0.022 & 0.005 & 0.005 & 0.005 & 0.006 & 0.006 & 0.007 & 0.006 & 0.005 & 0.016 & 0.019 & 0.023 & 0.010 & 0.011 & 0.012 & 0.011 \\
\hline Average & 0.020 & 0.022 & 0.005 & 0.005 & 0.005 & 0.006 & 0.006 & 0.007 & 0.006 & 0.005 & 0.016 & 0.019 & 0.023 & 0.010 & 0.011 & 0.012 & 0.011 \\
\hline
\end{tabular}

Sharpe ratios. We now consider the performance of portfolio policies in terms of risk-adjusted portfolio returns measured by the SR. In order to take into account the impact of portfolio turnovers reported in Table 3 we compute SR considering portfolio returns net of transaction costs of $0 \mathrm{bp}, 20 \mathrm{bp}$, and $50 \mathrm{bp}$. In each case, we indicate with an asterisk the instances in which the SR is statistically different with respect to the one obtained with the static covariance model PARA according to the bootstrap test proposed in Ledoit \& Wolf (2008) at the level of $5 \%$.

Table 4 reports the $\mathrm{SR}$ in the absence of transaction costs. We observe a pattern similar to that of the average gross returns reported in Table 1, with the specifications EWMA, ORE, and VECH displaying higher figures than the ones obtained with the static benchmark specification as well as to other dynamic counterparts when compared within the same portfolio selection policy. We also find that there is no substantial difference in the risk-adjusted performance of the portfolios obtained with the alternative static covariance models.

We report in Table 5 the SR when transaction costs of 20 bp are taken into account in the computation of risk-adjusted returns. This level of transaction costs is consistent with the estimate of French (2008), who finds 
Table 4: Sharpe ratios in the absence of transaction costs

The table reports the portfolio SR obtained by the each portfolio policy alongside each covariance model over the out-ofsample period. Figures are reported in percentages. The level of trasaction costs is $0 \mathrm{bp}$. The computation of portfolio returns net of transaction costs is described in Section 4. The portfolio policies are described in Section 2 whereas the covariance models are described in Section 3. The description of each acronym for the portfolio polices and for the covariance models are reported in Tables 10 and 11. Asterisks indicates the instances in which the SR is statistically different with respect to the one obtained with the static covariance model PARA according to the bootstrap test proposed in Ledoit \& Wolf (2008) at the level of $5 \%$

\begin{tabular}{|c|c|c|c|c|c|c|c|c|c|c|c|c|c|c|c|c|c|}
\hline Strategy & MiVU & $\mathrm{MeVU}$ & MiVC & $\mathrm{MeVC}$ & NCMV2.5 & NCMV5 & NCMV10 & VT1 & VT2 & VT4 & RwRS1 & RwRS2 & RwRS4 & RwR4F1 & RwR4F2 & RwR4F4 & Average \\
\hline \multicolumn{18}{|c|}{ Dynamic covariance models } \\
\hline EWMA & 0.103 & 0.107 & $0.101^{*}$ & $0.103^{*}$ & $0.104^{*}$ & $0.107^{*}$ & $0.111^{*}$ & 0.069 & $0.081^{*}$ & $0.090^{*}$ & $0.073^{*}$ & $0.092^{*}$ & $0.106^{*}$ & 0.061 & $0.065^{*}$ & 0.068 & 0.090 \\
\hline ORE & 0.099 & $0.100^{*}$ & 0.086 & 0.087 & 0.088 & 0.091 & 0.095 & $0.069^{*}$ & $0.078^{*}$ & $0.080^{*}$ & $0.070^{*}$ & $0.086^{*}$ & 0.096 & $0.060^{*}$ & $0.062^{*}$ & 0.065 & 0.082 \\
\hline VECH & 0.106 & 0.111 & $0.103^{*}$ & $0.106^{*}$ & $0.106^{*}$ & $0.108^{*}$ & $0.113^{*}$ & 0.069 & $0.081 *$ & $0.087^{*}$ & $0.071^{*}$ & $0.087^{*}$ & 0.102 & $0.062^{*}$ & $0.066^{*}$ & $0.072^{*}$ & 0.091 \\
\hline OGARCH & 0.094 & 0.093 & 0.078 & 0.078 & 0.080 & 0.081 & 0.083 & 0.066 & 0.073 & 0.073 & 0.064 & 0.071 & 0.083 & 0.057 & 0.058 & 0.061 & 0.075 \\
\hline $\mathrm{CCC}$ & 0.088 & 0.088 & 0.087 & 0.087 & 0.089 & 0.091 & 0.092 & 0.067 & 0.078 & 0.086 & 0.067 & 0.076 & 0.082 & 0.060 & 0.062 & 0.065 & 0.079 \\
\hline DCC & 0.039 & 0.036 & 0.100 & 0.101 & 0.103 & 0.105 & 0.108 & 0.067 & 0.078 & 0.086 & 0.067 & 0.076 & 0.082 & 0.060 & 0.062 & 0.065 & 0.077 \\
\hline DECO & 0.097 & 0.097 & 0.084 & 0.084 & 0.085 & 0.086 & 0.086 & 0.067 & 0.078 & 0.086 & 0.067 & 0.076 & 0.082 & 0.060 & 0.062 & 0.065 & 0.079 \\
\hline ASYDCC & $0.112^{*}$ & $0.111^{*}$ & $0.101^{*}$ & $0.102^{*}$ & $0.103^{*}$ & $0.106^{*}$ & $0.110^{*}$ & 0.061 & 0.067 & 0.078 & 0.063 & 0.073 & 0.078 & 0.054 & 0.054 & 0.054 & 0.083 \\
\hline Average & 0.092 & 0.093 & 0.093 & 0.093 & 0.095 & 0.097 & 0.100 & 0.067 & 0.077 & 0.083 & 0.068 & 0.080 & 0.089 & 0.059 & 0.061 & 0.064 & 0.082 \\
\hline \multicolumn{18}{|c|}{ Static covariance models } \\
\hline CORR & 0.078 & 0.078 & 0.076 & 0.076 & 0.078 & 0.080 & 0.083 & 0.066 & 0.073 & 0.072 & 0.066 & 0.075 & 0.087 & 0.057 & 0.057 & 0.056 & 0.072 \\
\hline $\begin{array}{l}\text { PARA } \\
\text { PAT }\end{array}$ & 0.079 & 0.078 & 0.077 & 0.077 & 0.080 & 0.081 & 0.084 & 0.066 & 0.072 & 0.072 & 0.065 & 0.075 & 0.087 & 0.057 & 0.056 & 0.056 & 0.073 \\
\hline MARKET & 0.078 & 0.078 & 0.077 & 0.077 & 0.079 & 0.081 & 0.084 & 0.066 & 0.073 & 0.072 & 0.066 & 0.075 & 0.087 & 0.057 & 0.057 & 0.056 & 0.073 \\
\hline Average & 0.078 & 0.078 & 0.077 & 0.077 & 0.079 & 0.081 & 0.084 & 0.066 & 0.072 & 0.072 & 0.065 & 0.075 & 0.087 & 0.057 & 0.057 & 0.056 & 0.073 \\
\hline
\end{tabular}

that Americans spend 21 basis points (bp) in total trading, as a fraction of the total portfolio. We observe that dynamic covariance specifications delivered higher SR in comparison to those obtained with static covariance models in very few instances. In most of the cases, the portfolios obtained with static covariance models outperformed those obtained with dynamic counterparts. This sharp decrease in risk-adjusted performance net of transaction costs obtained with dynamic covariance models is mostly explained by the presence of a much higher turnover when compared to static covariance models. In fact, we observe that many SR obtained with dynamic models turned out to the negative once an intermediate level of transaction costs are taken into account. In constrast, the SR of portfolio policies obtained with static covariance models were little affected by the presence of transaction costs.

\section{Table 5: Sharpe ratios based on portfolio returns under transaction costs of $20 \mathrm{bp}$}

The table reports the portfolio SR obtained by the each portfolio policy alongside each covariance model over the out-ofsample period. Figures are reported in percentages. The level of trasaction costs is 20 bp. The computation of portfolio returns net of transaction costs is described in Section 4. The portfolio policies are described in Section 2 whereas the covariance models are described in Section 3. The description of each acronym for the portfolio polices and for the covariance models are reported in Tables 10 and 11. Asterisks indicates the instances in which the SR is statistically different with respect to the one obtained with the static covariance model PARA according to the bootstrap test proposed in Ledoit \& Wolf (2008) at the level of $5 \%$

\begin{tabular}{|c|c|c|c|c|c|c|c|c|c|c|c|c|c|c|c|c|c|}
\hline Strategy & MiVU & $\mathrm{MeVU}$ & MiVC & $\mathrm{MeVC}$ & NCMV2.5 & NCMV5 & NCMV10 & VT1 & VT2 & VT4 & RwRS1 & RwRS2 & RwRS4 & RwR4F1 & RwR4F2 & RwR4F4 & Average \\
\hline \multicolumn{18}{|c|}{ Dynamic covariance models } \\
\hline EWMA & $0.018^{*}$ & $0.020^{*}$ & 0.081 & 0.082 & 0.083 & 0.084 & 0.087 & 0.067 & 0.075 & 0.078 & $0.069^{*}$ & $0.086^{*}$ & 0.099 & 0.059 & 0.060 & 0.060 & 0.069 \\
\hline ORE & 0.072 & 0.073 & 0.081 & 0.082 & 0.082 & 0.085 & 0.088 & $0.068^{*}$ & $0.076^{*}$ & 0.077 & $0.068^{*}$ & $0.083^{*}$ & 0.093 & 0.059 & 0.061 & 0.063 & 0.076 \\
\hline VECH & $-0.024^{*}$ & $-0.021^{*}$ & 0.064 & 0.067 & 0.065 & 0.065 & 0.066 & 0.064 & 0.068 & 0.061 & 0.065 & 0.076 & 0.086 & 0.057 & 0.056 & 0.054 & 0.054 \\
\hline OGARCH & 0.080 & 0.078 & $0.070^{*}$ & $0.070^{*}$ & $0.072^{*}$ & $0.073^{*}$ & $0.074^{*}$ & 0.064 & 0.071 & 0.070 & 0.061 & 0.067 & 0.076 & 0.056 & 0.055 & 0.056 & 0.068 \\
\hline $\mathrm{CCC}$ & $-0.030^{*}$ & $-0.027^{*}$ & $0.028^{*}$ & $0.027^{*}$ & $0.027^{*}$ & $0.026^{*}$ & $0.023^{*}$ & $0.057^{*}$ & $0.051^{*}$ & $0.030^{*}$ & $0.056^{*}$ & $0.053^{*}$ & $0.043^{*}$ & $0.051^{*}$ & $0.043^{*}$ & $0.029^{*}$ & 0.030 \\
\hline DCC & $-0.252^{*}$ & $-0.247^{*}$ & $0.020^{*}$ & $0.022^{*}$ & $0.020^{*}$ & $0.019^{*}$ & $0.016^{*}$ & $0.057^{*}$ & $0.051^{*}$ & $0.030^{*}$ & $0.056^{*}$ & $0.053^{*}$ & $0.043^{*}$ & $0.051 *$ & $0.043^{*}$ & $0.029^{*}$ & 0.001 \\
\hline DECO & $0.023^{*}$ & $0.024^{*}$ & $0.027^{*}$ & $0.026^{*}$ & $0.025^{*}$ & $0.024^{*}$ & $0.021^{*}$ & $0.057^{*}$ & $0.051^{*}$ & $0.030^{*}$ & $0.056^{*}$ & $0.053^{*}$ & $0.043^{*}$ & $0.051^{*}$ & $0.043^{*}$ & $0.029^{*}$ & 0.036 \\
\hline ASYDCC & $-0.008^{*}$ & $-0.004^{*}$ & $0.055^{*}$ & 0.059 & $0.056^{*}$ & $0.056^{*}$ & $0.058^{*}$ & $0.056^{*}$ & $0.057^{*}$ & $0.051^{*}$ & 0.058 & 0.064 & 0.071 & $0.050^{*}$ & $0.045^{*}$ & $0.038^{*}$ & 0.048 \\
\hline Average & -0.015 & -0.013 & 0.053 & 0.054 & 0.054 & 0.054 & 0.054 & 0.061 & 0.062 & 0.053 & 0.061 & 0.067 & 0.069 & 0.054 & 0.051 & 0.045 & 0.048 \\
\hline \multicolumn{18}{|c|}{ Static covariance models } \\
\hline CORR & 0.073 & 0.072 & 0.076 & 0.076 & 0.078 & 0.080 & 0.082 & 0.065 & 0.072 & 0.072 & 0.063 & 0.072 & 0.083 & 0.057 & 0.056 & 0.056 & 0.071 \\
\hline PARA & 0.073 & 0.072 & 0.077 & 0.077 & 0.079 & 0.081 & 0.084 & 0.065 & 0.072 & 0.072 & 0.063 & 0.071 & 0.083 & 0.056 & 0.056 & 0.055 & 0.071 \\
\hline MARKET & 0.072 & 0.071 & 0.077 & 0.077 & 0.079 & 0.080 & 0.083 & 0.065 & 0.072 & 0.072 & 0.063 & 0.072 & 0.083 & 0.057 & 0.056 & 0.056 & 0.071 \\
\hline Average & 0.073 & 0.072 & 0.077 & 0.077 & 0.079 & 0.080 & 0.083 & 0.065 & 0.072 & 0.072 & 0.063 & 0.072 & 0.083 & 0.057 & 0.056 & 0.055 & 0.071 \\
\hline
\end{tabular}

Finally, we report in Table 6 the SR when transaction costs of $50 \mathrm{bp}$ are taken into account, which corresponds to a scenario of high transaction costs. The results are similar to those reported in Table 5. The figures reveal 
that dynamic covariance models outperformed static ones in only three instances (RwRS1 and RwRS2 with EWMA and ORE specifications). In all remaining cases, we find that the risk-adjusted performance of portfolios obtained with dynamic covariance models are substantially affected by the presence of high transaction costs and underperformed those obtained with static models.

\section{Table 6: Sharpe ratios based on portfolio returns under transaction costs of $50 \mathrm{bp}$}

The table reports the portfolio SR obtained by the each portfolio policy alongside each covariance model over the out-of sample period. Figures are reported in percentages. The level of trasaction costs is $50 \mathrm{bp}$. The computation of portfolio returns net of transaction costs is described in Section 4. The portfolio policies are described in Section 2 whereas the covariance models are described in Section 3. The description of each acronym for the portfolio polices and for the covariance models are reported in Tables 10 and 11. Asterisks indicates the instances in which the SR is statistically different with respect to the one obtained with the static covariance model PARA according to the bootstrap test proposed in Ledoit \& Wolf (2008) at the level of $5 \%$

\begin{tabular}{|c|c|c|c|c|c|c|c|c|c|c|c|c|c|c|c|c|c|}
\hline Strategy & MiVU & $\mathrm{MeVU}$ & MiVC & $\mathrm{MeVC}$ & NCMV2.5 & NCMV5 & NCMV10 & VT1 & VT2 & VT4 & RwRS1 & RwRS2 & RwRS4 & RwR4F1 & RwR4F2 & RwR4F4 & Average \\
\hline \multicolumn{18}{|c|}{ Dynamic covariance models } \\
\hline EWMA & $-0.110^{*}$ & $-0.108^{*}$ & $0.048^{*}$ & $0.050^{*}$ & $0.049^{*}$ & $0.049^{*}$ & $0.049^{*}$ & 0.062 & 0.065 & 0.059 & 0.062 & $0.075^{*}$ & 0.087 & 0.054 & 0.051 & 0.045 & 0.037 \\
\hline ORE & $0.030^{*}$ & $0.031^{*}$ & 0.071 & 0.072 & 0.072 & 0.074 & 0.076 & 0.065 & 0.073 & 0.071 & $0.063^{*}$ & $0.076^{*}$ & 0.087 & 0.056 & 0.057 & 0.057 & 0.064 \\
\hline VECH & $-0.211^{*}$ & $-0.210^{*}$ & $0.003^{*}$ & $0.007^{*}$ & $0.001^{*}$ & $0.000^{*}$ & $-0.005^{*}$ & $0.054^{*}$ & $0.046^{*}$ & $0.021^{*}$ & 0.055 & 0.057 & 0.061 & $0.047^{*}$ & $0.038^{*}$ & $0.023^{*}$ & -0.001 \\
\hline OGARCH & 0.058 & 0.056 & $0.057^{*}$ & $0.057^{*}$ & $0.058^{*}$ & $0.059^{*}$ & $0.058^{*}$ & 0.061 & $0.066^{*}$ & $0.064^{*}$ & 0.055 & $0.058^{*}$ & $0.065^{*}$ & 0.052 & 0.049 & 0.046 & 0.057 \\
\hline CCC & $-0.206^{*}$ & $-0.199^{*}$ & $-0.064^{*}$ & $-0.064^{*}$ & $-0.068^{*}$ & $-0.072^{*}$ & $-0.081^{*}$ & $0.038^{*}$ & $0.008^{*}$ & $-0.055^{*}$ & $0.038^{*}$ & $0.015^{*}$ & $-0.016^{*}$ & $0.035 *$ & $0.011^{*}$ & $-0.028^{*}$ & -0.044 \\
\hline DCC & $-0.623^{*}$ & $-0.614^{*}$ & $-0.100^{*}$ & $-0.096^{*}$ & $-0.104^{*}$ & $-0.109^{*}$ & $-0.120^{*}$ & $0.038^{*}$ & $0.008^{*}$ & $-0.055^{*}$ & $0.038^{*}$ & $0.015^{*}$ & $-0.016^{*}$ & $0.035^{*}$ & $0.011^{*}$ & $-0.028^{*}$ & -0.108 \\
\hline DECO & $-0.089^{*}$ & $-0.086^{*}$ & $-0.061^{*}$ & $-0.062^{*}$ & $-0.067^{*}$ & $-0.071^{*}$ & $-0.078^{*}$ & $0.038^{*}$ & $0.008^{*}$ & $-0.055^{*}$ & $0.038^{*}$ & $0.015^{*}$ & $-0.016^{*}$ & $0.035^{*}$ & $0.011^{*}$ & $-0.028^{*}$ & -0.029 \\
\hline ASYDCC & $-0.186^{*}$ & $-0.173^{*}$ & $-0.016^{*}$ & $-0.008^{*}$ & $-0.017^{*}$ & $-0.019^{*}$ & $-0.022^{*}$ & $0.048^{*}$ & $0.038^{*}$ & $0.008^{*}$ & $0.048^{*}$ & $0.049^{*}$ & 0.057 & $0.042^{*}$ & $0.031 *$ & $0.012 *$ & -0.007 \\
\hline Average & -0.167 & $\begin{array}{c}-0.163 \\
\end{array}$ & -0.008 & -0.006 & -0.009 & -0.011 & -0.015 & 0.051 & 0.039 & 0.007 & 0.049 & 0.045 & 0.039 & 0.044 & 0.032 & 0.012 & -0.004 \\
\hline \multicolumn{18}{|c|}{ Static covariance models } \\
\hline CORR & 0.064 & 0.062 & 0.074 & 0.074 & 0.076 & 0.077 & 0.079 & 0.063 & 0.070 & 0.070 & 0.058 & 0.065 & 0.076 & 0.054 & 0.053 & 0.052 & 0.067 \\
\hline PARA & 0.064 & 0.062 & 0.075 & 0.075 & 0.077 & 0.079 & 0.081 & 0.063 & 0.070 & 0.070 & 0.058 & 0.065 & 0.076 & 0.054 & 0.052 & 0.051 & 0.067 \\
\hline MARKET & 0.063 & 0.061 & 0.075 & 0.075 & 0.077 & 0.078 & 0.080 & 0.063 & 0.070 & 0.070 & 0.058 & 0.065 & 0.076 & 0.054 & 0.053 & 0.052 & 0.067 \\
\hline Average & 0.064 & 0.062 & 0.075 & 0.075 & 0.077 & 0.078 & 0.080 & 0.063 & 0.070 & 0.070 & 0.058 & 0.065 & 0.076 & 0.054 & 0.053 & 0.052 & 0.067 \\
\hline
\end{tabular}

It is also worth analyzing the comparative performance in terms of risk-adjusted returns among alternative portfolio policies when transaction costs are taken into account. The results reported in Tables 4 to 6 leave three key messages. First, constrained versions of the mean-variance policies performed better than the unconstrained ones. This result is in line with those reported in Jagannathan \& Ma (2003) and in DeMiguel et al. (2009a). Second, when adopting dynamic covariance models, we find that portfolio policies that ignore off-diagonal covariance elements such as the VT and RwR policies of Kirby \& Ostdiek (2012) perform better than those than consider the full covariance structure. This result suggests that the estimation error in off-diagonal covariance elements plays an important role in the performance of portfolio policies that rely on this type of covariance specifications. Therefore, if the investor has an a priori preference for a given dynamic covariance specification, than he or she would be better off by adopting portfolio policies such as the VT and RwR policy in detriment of mean-variance policies. Third, we find that both mean-variance and VT-RwR policies performed similarly when considering static covariance specifications.

Performance fee for a risk-averse investor with quadratic utility. We report in Tables 7, 8 , and 9 the annualized performance fee that a risk-averse investor with quadratic utility and risk aversion coefficient $\gamma=1$ adopting a given portfolio policy is willing to pay in order to employ the PARA static covariance specification of Ledoit \& Wolf (2004) under the presence of 0 bp, 20 bp, and 50 bp transaction costs, respectively. Negative (positive) figures suggest that the pair portfolio policy/covariance model outperform (underperform) the pair portfolio policy/PARA covariance specification. The results are reassuring and corroborate to a large extent 
those reported in Tables 1 to 6. We find that under the absence of transaction costs, the investor is willing to pay an average of 28 bp per year on average across portfolio policies and covariance specifications in order to adopt dynamic covariance models. This result is expected, since dynamic models outperformed static counterparts in terms of risk-adjusted returns in the absence of transaction costs. However, we observe the opposite result once we consider the impact of transaction costs. The results in Table 8 reveal that in the presence of transaction costs of $20 \mathrm{bp}$, the investor is willing to pay an average annual fee of $368 \mathrm{bp}$ in order to switch to the PARA static covariance specification. This figure is further increased to an average of $966 \mathrm{bp}$ when the level of transaction costs is $50 \mathrm{bp}$.

Table 7: Performance fee to switch to the static PARA covariance specification in the absence of transaction costs

The table reports the performance fee that a risk-averse investor with quadratic utility adopting a given portfolio policy is willing to pay in order to use the PARA static covariance specification of Ledoit \& Wolf (2004). Figures are reported in annualized basis points. The level of trasaction costs is $0 \mathrm{bp}$. The portfolio policies are described in Section 2 whereas the covariance models are described in Section 3. The description of each acronym for the portfolio polices and for the covariance models are reported in Tables 10 and 11.

\begin{tabular}{|c|c|c|c|c|c|c|c|c|c|c|c|c|c|c|c|c|c|}
\hline Strategy & MiVU & $\mathrm{MeVU}$ & MiVC & $\mathrm{MeVC}$ & NCMV2.5 & NCMV5 & NCMV10 & VT1 & VT2 & VT4 & RwRS1 & RwRS2 & RwRS4 & RwR4F1 & RwR4F2 & RwR4F4 & Average \\
\hline \multicolumn{18}{|c|}{ Dynamic covariance models } \\
\hline EWMA & 38 & 213 & -117 & -75 & -129 & -136 & -137 & -32 & -53 & -75 & -79 & -250 & -662 & -5 & 17 & 45 & -90 \\
\hline ORE & -41 & 37 & -60 & -54 & -65 & -72 & -83 & -20 & -28 & -30 & -67 & -233 & -625 & -8 & -4 & 7 & -84 \\
\hline VECH & 81 & 227 & -23 & -9 & -30 & -27 & -17 & -17 & -21 & -28 & -30 & -84 & -265 & -8 & -5 & -25 & -18 \\
\hline OGARCH & -16 & -37 & -66 & -62 & -64 & -56 & -41 & -2 & -15 & -28 & 0 & 0 & 35 & 1 & -2 & 0 & -22 \\
\hline $\mathrm{CCC}$ & -75 & 3 & -83 & 15 & -82 & -76 & -61 & -9 & -43 & -104 & 27 & 64 & 74 & 37 & 65 & 75 & -11 \\
\hline DCC & 681 & 3348 & -248 & -243 & -249 & -237 & -216 & -9 & -43 & -104 & 27 & 64 & 74 & 37 & 65 & 75 & 189 \\
\hline DECO & -68 & -51 & -223 & -186 & -209 & -193 & -166 & -9 & -43 & -104 & 27 & 64 & 74 & 37 & 65 & 75 & -57 \\
\hline ASYDCC & -88 & 35 & -174 & -146 & -182 & -181 & -179 & -9 & -47 & -125 & -57 & -264 & -802 & 10 & 26 & 42 & -134 \\
\hline Average & 64 & 472 & -124 & -95 & -126 & -122 & -113 & -13 & -37 & -75 & -19 & -80 & -262 & 13 & 28 & 37 & -28 \\
\hline \multicolumn{18}{|c|}{ Static covariance models } \\
\hline CORR & 13 & 11 & 2 & 1 & 4 & 9 & 11 & 1 & 3 & 4 & 5 & 16 & 42 & 1 & 1 & -1 & 8 \\
\hline MARKET & 7 & 12 & -1 & -1 & 2 & 4 & 5 & 1 & 3 & 4 & 5 & 16 & 42 & 1 & 1 & -1 & 6 \\
\hline Average & 10 & 11 & 0 & 0 & 3 & 7 & 8 & 1 & 3 & 4 & 5 & 16 & 42 & 1 & 1 & -1 & 7 \\
\hline
\end{tabular}

Table 8: Performance fee to switch to the static PARA covariance specification in the presence of 20 bp transaction costs

The table reports the performance fee that a risk-averse investor with quadratic utility adopting a given portfolio policy is willing to pay in order to use the PARA static covariance specification of Ledoit \& Wolf (2004). Figures are reported in annualized basis points. The level of transaction costs is $20 \mathrm{bp}$. The portfolio policies are described in Section 2 whereas the covariance models are described in Section 3. The description of each acronym for the portfolio polices and for the covariance models are reported in Tables 10 and 11.

\begin{tabular}{|c|c|c|c|c|c|c|c|c|c|c|c|c|c|c|c|c|c|}
\hline Strategy & MiVU & $\mathrm{MeVU}$ & MiVC & $\mathrm{MeVC}$ & NCMV2.5 & NCMV5 & NCMV10 & VT1 & VT2 & VT4 & RwRS1 & RwRS2 & RwRS4 & RwR4F1 & RwR4F2 & RwR4F4 & Average \\
\hline \multicolumn{18}{|c|}{ Dynamic covariance models } \\
\hline EWMA & 726 & 963 & 49 & 88 & 41 & 38 & 48 & -13 & -6 & 20 & -66 & -223 & -636 & 14 & 62 & 125 & 77 \\
\hline ORE & 127 & 209 & -19 & -15 & -24 & -30 & -38 & -17 & -20 & -10 & -67 & -235 & -650 & -5 & 4 & 22 & -48 \\
\hline VECH & 1216 & 1504 & 310 & 314 & 311 & 324 & 356 & 35 & 96 & 191 & 10 & -1 & -144 & 43 & 105 & 151 & 301 \\
\hline OGARCH & 53 & 23 & -6 & -3 & -2 & 10 & 31 & 6 & 2 & -8 & 4 & 7 & 45 & 9 & 17 & 24 & 13 \\
\hline $\mathrm{CCC}$ & 875 & 958 & 439 & 527 & 455 & 474 & 513 & 107 & 215 & 393 & 129 & 282 & 434 & 144 & 294 & 462 & 419 \\
\hline DCC & 5239 & 16676 & 485 & 462 & 500 & 530 & 587 & 107 & 215 & 393 & 129 & 282 & 434 & 144 & 294 & 462 & 1684 \\
\hline $\mathrm{DECO}$ & 531 & 540 & 282 & 307 & 307 & 333 & 373 & 107 & 215 & 393 & 129 & 282 & 434 & 144 & 294 & 462 & 321 \\
\hline ASYDCC & 881 & 1027 & 233 & 244 & 234 & 245 & 268 & 41 & 69 & 134 & -19 & -199 & -795 & 55 & 132 & 233 & 174 \\
\hline Average & 1206 & 2737 & 222 & 240 & 228 & 240 & 267 & 47 & 98 & 188 & 31 & 24 & -110 & 69 & 150 & 243 & 368 \\
\hline \multicolumn{18}{|c|}{ Static covariance models } \\
\hline CORR & 9 & 6 & 1 & 0 & 4 & 8 & 10 & 1 & 3 & 3 & 5 & 16 & 44 & 1 & 1 & -1 & 7 \\
\hline MARKET & 7 & 12 & -1 & -2 & 1 & 4 & 4 & 1 & 3 & 3 & 5 & 16 & 44 & 1 & 1 & -1 & 6 \\
\hline Average & 8 & 9 & 0 & -1 & 3 & 6 & 7 & 1 & 3 & 3 & 5 & 16 & 44 & 1 & 1 & -1 & 7 \\
\hline
\end{tabular}


Table 9: Performance fee to switch to the static PARA covariance specification in the presence of 50 bp transaction costs

The table reports the performance fee that a risk-averse investor with quadratic utility adopting a given portfolio policy is willing to pay in order to use the PARA static covariance specification of Ledoit \& Wolf (2004). Figures are reported in annualized basis points. The level of transaction costs is $50 \mathrm{bp}$. The portfolio policies are described in Section 2 whereas the covariance models are described in Section 3. The description of each acronym for the portfolio polices and for the covariance models are reported in Tables 10 and 11.

\begin{tabular}{|c|c|c|c|c|c|c|c|c|c|c|c|c|c|c|c|c|c|}
\hline Strategy & MiVU & $\mathrm{MeVU}$ & MiVC & $\mathrm{MeVC}$ & NCMV2.5 & NCMV5 & NCMV10 & VT1 & VT2 & VT4 & RwRS1 & RwRS2 & RwRS4 & RwR4F1 & RwR4F2 & RwR4F4 & Average \\
\hline \multicolumn{18}{|c|}{ Dynamic covariance models } \\
\hline EWMA & 726 & 963 & 49 & 88 & 41 & 38 & 48 & -13 & -6 & 20 & -66 & -223 & -636 & 14 & 62 & 125 & 77 \\
\hline ORE & 127 & 209 & -19 & -15 & -24 & -30 & -38 & -17 & -20 & -10 & -67 & -235 & -650 & -5 & 4 & 22 & -48 \\
\hline VECH & 1216 & 1504 & 310 & 314 & 311 & 324 & 356 & 35 & 96 & 191 & 10 & -1 & -144 & 43 & 105 & 151 & 301 \\
\hline OGARCH & 53 & 23 & -6 & -3 & -2 & 10 & 31 & 6 & 2 & -8 & 4 & 7 & 45 & 9 & 17 & 24 & 13 \\
\hline $\mathrm{CCC}$ & 875 & 958 & 439 & 527 & 455 & 474 & 513 & 107 & 215 & 393 & 129 & 282 & 434 & 144 & 294 & 462 & 419 \\
\hline DCC & 5239 & 16676 & 485 & 462 & 500 & 530 & 587 & 107 & 215 & 393 & 129 & 282 & 434 & 144 & 294 & 462 & 1684 \\
\hline $\mathrm{DECO}$ & 531 & 540 & 282 & 307 & 307 & 333 & 373 & 107 & 215 & 393 & 129 & 282 & 434 & 144 & 294 & 462 & 321 \\
\hline ASYDCC & 881 & 1027 & 233 & 244 & 234 & 245 & 268 & 41 & 69 & 134 & -19 & -199 & -795 & 55 & 132 & 233 & 174 \\
\hline Average & 1206 & 2737 & 222 & 240 & 228 & 240 & 267 & 47 & 98 & 188 & 31 & 24 & -110 & 69 & 150 & 243 & 368 \\
\hline \multicolumn{18}{|c|}{ Static covariance models } \\
\hline CORR & 9 & 6 & 1 & 0 & 4 & 8 & 10 & 1 & 3 & 3 & 5 & 16 & 44 & 1 & 1 & -1 & 7 \\
\hline MARKET & 7 & 12 & -1 & -2 & 1 & 4 & 4 & 1 & 3 & 3 & 5 & 16 & 44 & 1 & 1 & -1 & 6 \\
\hline Average & 8 & 9 & 0 & -1 & 3 & 6 & 7 & 1 & 3 & 3 & 5 & 16 & 44 & 1 & 1 & -1 & 7 \\
\hline
\end{tabular}

\section{Concluding Remarks}

The literature still lacks consensus regarding what is the best way to model the covariance matrix of asset returns for high dimensional portfolio selection problems. This work adds to this discussion by comparing the most popular alternatives in a realistic scenario in which i) there exists many assets, ii) transaction costs are taken into account, and iii) there is frequent portfolio re-balancing. We find that in the absence of transaction costs, dynamic covariance models outperform static counterparts in terms of average gross returns as well as in terms of risk-adjusted returns. On average, Sharpe ratios obtained with dynamic covariance models are $12 \%$ higher than those obtained with static covariance models, and the pairwise differences are statistically significant in many instances. However, as we move to more realistic scenarios in which transaction costs are properly taken into account, we find that static covariance models clearly outperform in the vast majority of instances. Specifically, Sharpe rations based on portfolio returns net of transaction costs of 20 basis points (bp) are $48 \%$ higher when static covariance models are adopted in comparison to those obtained with dynamic models. This difference in risk adjusted performance becomes even higher when transaction costs of 50 bp are considered. A closer examination reveals that these differences in risk-adjusted performance are mainly driven by a much higher level of turnover obtained when dynamic covariance models are used to implement the portfolio policies. 


\section{Appendix}

Table 10: Portfolio selection policies and their respectrive acronyms

\begin{tabular}{ll}
\hline Acronym & Policy description \\
\hline MiVU & Unrestricted Minimum-variance Portfolio \\
MeVU & Unrestricted Mean-variance Portfolio \\
MiVC & Restricted Minimum-variance Portfolio \\
MeVC & Restricted Mean-variance Portfolio \\
NCMV2.5 & Mean-variance with Norm Restriction $(\delta=2.5 \%)$ \\
NCMV5 & Mean-variance with Norm Restriction $(\delta=5 \%)$ \\
NCMV10 & Mean-variance with Norm Restriction $(\delta=10 \%)$ \\
VT1 & Volatility timing $(\eta=1)$ \\
VT2 & Volatility timing $(\eta=2)$ \\
VT4 & Volatility timing $(\eta=4)$ \\
RwRS1 & Reward-to-risk $(\eta=1)$ \\
RwRS2 & Reward-to-risk $(\eta=2)$ \\
RwRS4 & Reward-to-risk $(\eta=4)$ \\
RwR4F1 & Reward-to-risk with 4-factor model $(\eta=1)$ \\
RwR4F2 & Reward-to-risk with 4-factor model $(\eta=2)$ \\
RwR4F4 & Reward-to-risk with 4-factor model $(\eta=4)$ \\
\hline &
\end{tabular}

Table 11: Covariance models and their respective acronyms

\begin{tabular}{ll}
\hline Acronym & Covariance model description \\
\hline CORR & Shrinkage method with constant correlation target \\
PARA & Shrinkage method with identity matrix target \\
MARKET & Shrinkage method with market model target \\
EWMA & Exponentially Weighted Moving Average \\
ORE & Optimal Rolling Estimator \\
VECH & Scalar VECH \\
OGARCH & Ortoghonal GARCH \\
CCC & Constant Conditional Correlation \\
DCC & Dynamic Conditional Correlation \\
ASYDCC & Asymmetric Dynamic Conditional Correlation \\
DECO & Dynamic Equicorrelation \\
\hline
\end{tabular}


Table 12: Descriptive statistics

\begin{tabular}{|c|c|c|c|c|c|}
\hline Ticker & Company & Mean & Variance & Asymmetry & Kurtosis \\
\hline AAPL & Apple Inc. & 0.159 & 5.445 & -0.092 & 7.968 \\
\hline $\mathrm{BAC}$ & Bank of America Corp & -0.026 & 13.051 & -0.307 & 24.099 \\
\hline MSFT & Microsoft Corporation & 0.024 & 2.881 & 0.082 & 14.252 \\
\hline INTC & Intel Corporation & 0.002 & 3.654 & -0.308 & 8.000 \\
\hline $\mathrm{CSCO}$ & Cisco Systems. Inc. & -0.001 & 4.025 & -0.404 & 13.272 \\
\hline GE & General Electric Company & 0.009 & 3.783 & -0.012 & 14.653 \\
\hline $\mathrm{F}$ & Ford Motor Company & 0.004 & 8.980 & -0.075 & 18.679 \\
\hline PFE & Pfizer Inc. & 0.010 & 2.277 & -0.357 & 11.060 \\
\hline ORCL & Oracle Corporation & 0.044 & 3.583 & -0.042 & 8.336 \\
\hline WFC & Wells Fargo \& Co & 0.029 & 8.662 & 0.878 & 24.915 \\
\hline JPM & JPMorgan Chase \& Co. & 0.029 & 7.398 & 0.337 & 18.011 \\
\hline $\mathrm{C}$ & Citigroup Inc & -0.080 & 14.564 & -0.480 & 36.052 \\
\hline EMC & EMC Corporation & 0.026 & 4.166 & 0.194 & 7.124 \\
\hline $\mathrm{T}$ & AT\&T Inc. & 0.032 & 1.995 & 0.528 & 14.249 \\
\hline $\mathrm{XOM}$ & Exxon Mobil Corporation & 0.045 & 2.532 & 0.016 & 17.286 \\
\hline FCX & Freeport-McMoRan Inc & 0.038 & 10.390 & -0.497 & 8.497 \\
\hline CMCSA & Comcast Corporation & 0.039 & 3.827 & 0.042 & 16.210 \\
\hline HPQ & Hewlett-Packard Company & 0.013 & 4.413 & -0.481 & 15.320 \\
\hline $\mathrm{KO}$ & The Coca-Cola Co & 0.031 & 1.372 & 0.427 & 16.323 \\
\hline GILD & Gilead Sciences. Inc. & 0.093 & 4.032 & -0.126 & 9.436 \\
\hline QCOM & QUALCOMM. Inc. & 0.046 & 4.058 & 0.001 & 9.491 \\
\hline MS & Morgan Stanley & -0.010 & 12.801 & 1.635 & 53.171 \\
\hline EBAY & eBay Inc & 0.022 & 5.593 & -0.010 & 10.961 \\
\hline $\mathrm{VZ}$ & Verizon Communications Inc. & 0.037 & 1.897 & 0.303 & 11.446 \\
\hline HAL & Halliburton Company & 0.059 & 6.861 & -0.504 & 10.038 \\
\hline MRK & Merck \& Co.. Inc. & 0.019 & 3.291 & -2.412 & 46.201 \\
\hline WMT & Wal-Mart Stores. Inc. & 0.024 & 1.463 & 0.144 & 10.106 \\
\hline TXN & Texas Instruments Incorporated & 0.022 & 3.795 & -0.257 & 6.888 \\
\hline $\mathrm{ABT}$ & Abbott Laboratories & 0.038 & 1.642 & 0.089 & 9.418 \\
\hline HD & Home Depot Inc & 0.043 & 3.036 & 0.406 & 7.956 \\
\hline $\mathrm{COP}$ & ConocoPhillips & 0.054 & 3.748 & -0.457 & 10.739 \\
\hline MO & Altria Group Inc & 0.066 & 1.627 & -0.171 & 20.595 \\
\hline USB & U.S. Bancorp & 0.025 & 5.727 & -0.024 & 19.092 \\
\hline LOW & Lowe's Companies. Inc. & 0.029 & 3.911 & 0.287 & 7.734 \\
\hline JNJ & Johnson \& Johnson & 0.034 & 1.020 & 0.584 & 16.367 \\
\hline BMY & Bristol-Myers Squibb Co & 0.042 & 2.157 & 0.083 & 8.260 \\
\hline DIS & Walt Disney Co & 0.052 & 3.116 & 0.412 & 11.689 \\
\hline PG & Procter \& Gamble Co & 0.030 & 1.261 & -0.187 & 10.414 \\
\hline SLB & Schlumberger Limited. & 0.052 & 5.713 & -0.554 & 10.360 \\
\hline CVX & Chevron Corporation & 0.055 & 2.901 & 0.074 & 17.512 \\
\hline SBUX & Starbucks Corporation & 0.064 & 4.504 & 0.275 & 8.587 \\
\hline CVS & CVS Health Corp & 0.059 & 2.929 & -1.073 & 21.903 \\
\hline MDLZ & Mondelez International Inc & 0.032 & 1.606 & -0.122 & 8.323 \\
\hline $\mathrm{AXP}$ & American Express Company & 0.037 & 6.019 & 0.069 & 14.596 \\
\hline DOW & Dow Chemical Co & 0.017 & 5.462 & -0.433 & 11.118 \\
\hline UNH & UnitedHealth Group Inc. & 0.040 & 4.811 & 0.417 & 27.028 \\
\hline GS & Goldman Sachs Group Inc & 0.028 & 6.317 & 0.304 & 18.093 \\
\hline AMGN & Amgen. Inc. & 0.026 & 2.789 & 0.729 & 12.046 \\
\hline TWX & Time Warner Inc & 0.023 & 3.565 & -0.645 & 20.400 \\
\hline CAT & Caterpillar Inc. & 0.041 & 4.474 & -0.198 & 8.585 \\
\hline
\end{tabular}




\section{References}

Alexander, Carol. 2001. Orthogonal garch. Mastering risk, 2, 21-38.

Bauwens, Luc, Laurent, Sébastien, \& Rombouts, Jeroen VK. 2006. Multivariate GARCH models: a survey. Journal of applied econometrics, 21(1), 79-109.

Becker, Ralf, Clements, AE, Doolan, MB, \& Hurn, AS. 2014. Selecting volatility forecasting models for portfolio allocation purposes. International Journal of Forecasting.

Bollerslev, Tim. 1990. Modelling the coherence in short-run nominal exchange rates: a multivariate generalized ARCH model. The Review of Economics and Statistics, 498-505.

Bollerslev, Tim, Engle, Robert F, \& Wooldridge, Jeffrey M. 1988. A capital asset pricing model with timevarying covariances. The Journal of Political Economy, 116-131.

Cappiello, Lorenzo, Engle, Robert F, \& Sheppard, Kevin. 2006. Asymmetric dynamics in the correlations of global equity and bond returns. Journal of Financial econometrics, 4(4), 537-572.

Carhart, Mark M. 1997. On persistence in mutual fund performance. The Journal of finance, 52(1), 57-82.

Della Corte, Pasquale, Sarno, Lucio, \& Thornton, Daniel L. 2008. The expectation hypothesis of the term structure of very short-term rates: Statistical tests and economic value. Journal of Financial Economics, 89(1), $158-174$.

DeMiguel, Victor, Garlappi, Lorenzo, Nogales, Francisco J, \& Uppal, Raman. 2009a. A generalized approach to portfolio optimization: Improving performance by constraining portfolio norms. Management Science, 55(5), 798812.

DeMiguel, Victor, Garlappi, Lorenzo, \& Uppal, Raman. 2009b. Optimal versus naive diversification: How inefficient is the $1 / \mathrm{N}$ portfolio strategy? Review of Financial Studies, 22(5), 1915-1953.

DeMiguel, Victor, Nogales, Francisco J, \& Uppal, Raman. 2014. Stock return serial dependence and out-ofsample portfolio performance. Review of Financial Studies, 27(4), 1031-1073.

Engle, RoBert. 2002. Dynamic conditional correlation: A simple class of multivariate generalized autoregressive conditional heteroskedasticity models. Journal of Business \& Economic Statistics, 20(3), 339-350.

Engle, Robert, \& Colacito, Riccardo. 2006. Testing and valuing dynamic correlations for asset allocation. Journal of Business \&5 Economic Statistics, 24(2).

Engle, Robert, \& Kelly, Bryan. 2012. Dynamic equicorrelation. Journal of Business E Economic Statistics, 30(2), $212-228$.

Engle, Robert, \& Mezrich, Joseph. 1996. Garch for Groups: A round-up of recent developments in Garch techniques for estimating correlation. RISK, 9, 36-40.

Engle, Robert, \& Sheppard, Kevin. 2008. Evaluating the specification of covariance models for large portfolios. New York University, working paper.

Engle, Robert F, Shephard, Neil, \& Sheppard, Kevin. 2008. Fitting vast dimensional time-varying covariance models.

Fama, Eugene F, \& French, Kenneth R. 1992. The cross-section of expected stock returns. the Journal of Finance, 47(2), 427-465.

Fleming, Jeff, Kirby, Chris, \& Ostdiek, Barbara. 2001. The economic value of volatility timing. The Journal of Finance, 56(1), 329-352.

Fleming, Jeff, Kirby, Chris, \& Ostdiek, Barbara. 2003. The economic value of volatility timing using realized volatility. Journal of Financial Economics, 67(3), 473-509.

Foster, Dean P, \& Nelson, Daniel B. 1996. Continuous Record Asymptotics for Rolling Sample Variance Estimators. Econometrica, 64(1), 139-74.

French, Kenneth R. 2008. Presidential address: The cost of active investing. The Journal of Finance, 63(4), 15371573.

Hafner, Christian M, \& Reznikova, Olga. 2012. On the estimation of dynamic conditional correlation models. Computational Statistics \& Data Analysis, 56(11), 3533-3545. 
Han, Yufeng. 2006. Asset allocation with a high dimensional latent factor stochasticvolatility model. Review of Financial Studies, 19(1), 237-271.

Jagannathan, Ravi, \& Ma, Tongshu. 2003. Risk Reduction in Large Portfolios: Why Imposing the Wrong Constraints Helps. The Journal of Finance, 58(4), 1651-1684.

Kirby, Chris, \& Ostdiek, Barbara. 2012. It's all in the timing: simple active portfolio strategies that outperform naive diversification. Journal of Financial and Quantitative Analysis, 47(02), 437-467.

Kolm, Petter N, Tütüncü, Reha, \& Fabozzi, Frank J. 2014. 60 Years of portfolio optimization: Practical challenges and current trends. European Journal of Operational Research, 234(2), 356-371.

Ledoit, Oliver, \& Wolf, Michael. 2008. Robust performance hypothesis testing with the Sharpe ratio. Journal of Empirical Finance, 15(5), 850-859.

Ledoit, Olivier, \& Wolf, Michael. 2003a. Honey, I shrunk the sample covariance matrix. UPF Economics and Business Working Paper.

Ledoit, Olivier, \& Wolf, Michael. 2003b. Improved estimation of the covariance matrix of stock returns with an application to portfolio selection. Journal of empirical finance, 10(5), 603-621.

Ledoit, Olivier, \& Wolf, Michael. 2004. A well-conditioned estimator for large-dimensional covariance matrices. Journal of multivariate analysis, 88(2), 365-411.

Markowitz, Harry. 1952. Portfolio selection*. The journal of finance, 7(1), 77-91.

Merton, Robert C. 1980. On estimating the expected return on the market: An exploratory investigation. Journal of Financial Economics, 8(4), 323-361.

Olivares-Nadal, Alba V, \& DeMiguel, Victor. 2015. A Robust Perspective on Transaction Costs in Portfolio Optimization.

Pooter, Michiel de, Martens, Martin, \& Dijk, Dick van. 2008. Predicting the daily covariance matrix for s\&p 100 stocks using intraday data: But which frequency to use? Econometric Reviews, 27(1-3), 199-229.

Silvennoinen, Annastimna, \& Teräsvirta, Timo. 2009. Multivariate GARCH models. Pages 201-229 of: Handbook of financial time series. Springer.

Thornton, Daniel L, \& Valente, Giorgio. 2012. Out-of-sample predictions of bond excess returns and forward rates: An asset allocation perspective. Review of Financial Studies, 25(10), 3141-3168.

ZAfFaroni, PAOlo. 2008. Large-scale volatility models: theoretical properties of professionals' practice. Journal of Time Series Analysis, 29(3), 581-599. 